\title{
New algebras of functions on topological groups arising from $G$-spaces
}

\author{
by \\ E. Glasner (Tel-Aviv) and M. Megrelishvili (Ramat-Gan)
}

\begin{abstract}
For a topological group $G$ we introduce the algebra $S U C(G)$ of strongly uniformly continuous functions. We show that $S U C(G)$ contains the algebra $W A P(G)$ of weakly almost periodic functions as well as the algebras $L E(G)$ and $\operatorname{Asp}(G)$ of locally equicontinuous and Asplund functions respectively. For the Polish groups of order preserving homeomorphisms of the unit interval and of isometries of the Urysohn space of diameter 1, we show that $S U C(G)$ is trivial. We introduce the notion of fixed point on a class $\mathrm{P}$ of flows (P-fpp) and study in particular groups with the SUC-fpp. We study the Roelcke algebra ( $=U C(G)=$ right and left uniformly continuous functions) and SUC compactifications of the groups $S(\mathbb{N})$, of permutations of a countable set, and $H(C)$, of homeomorphisms of the Cantor set. For the first group we show that $W A P(G)=S U C(G)=U C(G)$ and also provide a concrete description of the corresponding metrizable (in fact Cantor) semitopological semigroup compactification. For the second group, in contrast, we show that $S U C(G)$ is properly contained in $U C(G)$. We then deduce that for this group $U C(G)$ does not yield a right topological semigroup compactification.
\end{abstract}

\section{Contents}

1. Introduction

2. Actions and $G$-compactifications 4

3. Cyclic $G$-systems and point-universality $\quad 7$

4. Strong uniform continuity 10

5. SUC, homogeneity and the epimorphism problem 15

6. Representations of groups and $G$-spaces on Banach spaces 17

7. Dynamical complexity of functions 21

8. The group $H_{+}[0,1] \quad 27$

9. Matrix coefficient characterization of SUC and LE 30

10. Some conclusions about $H_{+}[0,1]$ and $\operatorname{Iso}\left(\mathbb{U}_{1}\right)$

11. Relative extreme amenability: SUC-fpp groups 40

2000 Mathematics Subject Classification: 37B05, 43A60, 54H20, 54H15.

Key words and phrases: Asplund function, fixed point property, $G$-compactification, $G$-space, locally equicontinuous, matrix coefficient, right topological semigroup compactification, strongly uniformly continuous.

Research partially supported by BSF (Binational USA-Israel) grant no. 2006119. 
12. The Roelcke compactification of the group $S(\mathbb{N})$

1. Introduction. In this paper we introduce the property of strong uniform continuity (for short: SUC) of $G$-spaces and the associated notion of SUC functions. For every compact $G$-space $X$ the corresponding orbit maps $\widetilde{x}: G \rightarrow X, g \mapsto g x$, are right uniformly continuous for every $x \in X$. If all the maps $\{\widetilde{x}\}_{x \in X}$ are also left uniformly continuous then we say that $X$ is SUC. Every right uniformly continuous bounded real-valued function $f: G \rightarrow \mathbb{R}$ comes from some compact $G$-space $X$. That is, there exist a compact $G$-space $X$, a continuous function $F: X \rightarrow \mathbb{R}$, and a point $x_{0} \in X$ such that $f=F \circ \widetilde{x}_{0}$. We say that $f$ is SUC if it comes from a compact $G$-space $X$ which is SUC. We denote by $S U C(G)$ the corresponding class of functions on $G$. The class $S U C(G)$ forms a uniformly closed $G$ invariant subalgebra of the algebra $U C(G):=R U C(G) \cap L U C(G)$ of (right and left) uniformly continuous functions. Of course we have $S U C(G)=$ $U C(G)=R U C(G)=L U C(G)$ when $G$ is either discrete or abelian so that the notion of strong uniform continuity can be useful only when one deals with nonabelian nondiscrete topological groups. Mostly we will be interested in Polish non-locally compact large groups, but some of the questions we study are of interest in the locally compact case as well.

In our recent work [13] we investigated, among other topics, the algebras of locally equicontinuous functions, $L E(G)$, and Asplund functions, $\operatorname{Asp}(G)$, on a topological group $G$. The inclusions $U C(G) \supset S U C(G) \supset L E(G) \supset$ $\operatorname{Asp}(G) \supset W A P(G)$ hold for an arbitrary topological group $G$. In the present article we provide a characterization of the elements of $S U C(G)$ and $L E(G)$ in terms of matrix coefficients for appropriate Banach representations of $G$ by linear isometries.

Intuitively the dynamical complexity of a function $f \in R U C(G)$ can be estimated by the topological complexity of the cyclic $G$-flow $X_{f}$ (the pointwise closure of the left $G$-orbit $\{g f\}_{g \in G}$ of $f$ ) treating it as a subset of the Banach space $R U C(G)$. This leads to a natural dynamical hierarchy (see Theorem 7.12) where $S U C(G)$ plays a basic role. In some sense $S U C(G)$ is the largest "nice subalgebra" of $U C(G)$. It turns out that $f \in S U C(G)$ iff $X_{f}$ is a subset of $U C(G)$. Moreover the algebra $S U C(G)$ is point-universal in the sense of [13] and any other point-universal subalgebra of $U C(G)$ is contained in $S U C(G)$. Recall that a $G$-algebra $\mathcal{A} \subset R U C(G)$ is point-universal if and only if the associated $G$-compactification $G \rightarrow G^{\mathcal{A}}$ is a right topological semigroup compactification.

As an application we conclude that the algebra $U C(G)$ is point-universal if and only if it coincides with the algebra $S U C(G)$ and that the corre- 
sponding Roelcke compactification $G \rightarrow G^{U C}$ is in general not a right topological semigroup compactification of $G$ (in contrast to the compactification $G \rightarrow G^{S U C}$ determined by the algebra $\left.S U C(G)\right)$.

For locally compact groups, $S U C(G)$ contains the subalgebra $C_{0}(G)$ consisting of the functions which vanish at infinity, and therefore determines the topology of $G$. The structure of $S U C(G)$-in contrast to $R U C(G)$ which is always huge for non-precompact groups - is "computable" for several large groups like: $H_{+}[0,1]$, Iso $\left(\mathbb{U}_{1}\right)$ (the isometry group of the Urysohn space of diameter one $\left.\mathbb{U}_{1}\right), U(H)$ (the unitary group on an infinite-dimensional Hilbert space), $S_{\infty}=S(\mathbb{N})$ (the Polish infinite symmetric group) and any noncompact connected simple Lie group with finite center (e.g., $\left.S L_{n}(\mathbb{R})\right)$. For instance, $S U C(G)=W A P(G)$ for $U(H), S_{\infty}$ and $S L_{n}(\mathbb{R})$. In the first case we use a result of Uspenskij [46] which identifies the Roelcke completion of $U(H)$ as the compact semigroup of contracting operators on the Hilbert space $H$. For $S_{\infty}$ see Section 12, and for $S L_{n}(\mathbb{R})$ this follows from an old result of Veech [49].

The group $H_{+}[0,1]$ of orientation preserving homeomorphisms of the closed unit interval, endowed with the compact-open topology, is a good test case in the class of "large" yet "computable" topological groups. See Section 8 for more details on this group. In particular recall the result from [28] which shows that $H_{+}[0,1]$ is WAP-trivial: Every weakly almost periodic function on $G:=H_{+}[0,1]$ is a constant. Equivalently, $G$ is reflexively trivial, that is, every continuous representation $G \rightarrow \operatorname{Iso}(V)$, where $V$ is a reflexive Banach space, is trivial.

Here we show that $G$ is even "SUC-trivial" - that is, the algebra $S U C(G)$ (and hence, also the algebras $L E(G)$ and $\operatorname{Asp}(G)$ ) consists only of constant functions - and that every continuous representation of $G$ into the group of linear isometries $\operatorname{Iso}(V)$ of an Asplund Banach space $V$ is trivial. Since in general $W A P(G) \subset \operatorname{Asp}(G)$ and since every reflexive Banach space is Asplund these results strengthen the main results of [28]. SUC-triviality implies that every adjoint continuous (see Section 6) representation is trivial for $H_{+}[0,1]$. The latter fact also follows from a recent unpublished result of Uspenskij (private communication).

From the WAP-triviality (equivalently, reflexive triviality) of $H_{+}[0,1]$ and results of Uspenskij about Iso $\left(\mathbb{U}_{1}\right)$ Pestov deduces in [39, Corollary 1.4] the fact that the group $\operatorname{Iso}\left(\mathbb{U}_{1}\right)$ is also WAP-trivial. Using a similar idea and the matrix coefficient characterization of SUC one can conclude that $\operatorname{Iso}\left(\mathbb{U}_{1}\right)$ is SUC-trivial. It is an open question whether the group $H\left([0,1]^{\omega}\right)$ is SUC-trivial (or WAP-trivial).

The above mentioned description of $S U C(G)$ and $L E(G)$ in terms of matrix coefficients (Section 9) is nontrivial. The proof is based on a dynamical modification of a well known interpolation technique of Davis, Figiel, Johnson and Pełczyński [7]. 
In Section 11 we introduce a notion of extreme amenability with respect to a class of flows. In particular, we examine extreme $S U C$-amenability and extreme $S U C$-amenable groups, which are those groups which have the fixed point property on compact SUC $G$-spaces. Several natural groups, like $S L_{2}(\mathbb{R}), S_{\infty}, H(C)$ (the homeomorphism group of the Cantor set), and $H_{+}(\mathbb{S})$, which fail to be extremely amenable, are however extremely SUC-amenable. In the last two sections we study the Roelcke and SUC compactifications of the groups $S_{\infty}$ and $H(C)$. For the first group we show that $W A P\left(S_{\infty}\right)=S U C\left(S_{\infty}\right)=U C\left(S_{\infty}\right)$ and also provide a concrete description of the corresponding metrizable (in fact Cantor) semitopological semigroup compactification. For the latter group $G:=H(C)$, in contrast, we have $S U C(G) \subsetneq U C(G)$, from which we deduce that the corresponding Roelcke compactification $G \rightarrow G^{U C}$ is not a right topological semigroup compactification of $G$.

Finally, let us note that although in this work we consider, for convenience, algebras of real-valued functions, it seems that there should be no difficulty in extending our definitions and results to the complex case.

2. Actions and $G$-compactifications. Unless explicitly stated otherwise, all spaces in this paper are at least Tikhonov. A (left) action of a topological group $G$ on a topological space $X$ is defined by a function $\pi$ : $G \times X \rightarrow X, \pi(g, x):=g x$, such that always $g_{1}\left(g_{2} x\right)=\left(g_{1} g_{2}\right) x$ and $e x=x$, where $e=e_{G}$ is the neutral element of $G$. Every $x \in X$ defines an orbit map $\widetilde{x}: G \rightarrow X, g \mapsto g x$. Also every $g \in G$ induces a $g$-translation $\pi^{g}: X \rightarrow X$, $x \mapsto g x$. If the action $\pi$ is continuous then we say that $X$ is a $G$-space (or a $G$-system or a $G$-flow). Sometimes we denote it as a pair $(G, X)$. If the orbit $G x_{0}$ of $x_{0}$ is dense in $X$ for some $x_{0} \in X$ then the $G$-space $X$ is point transitive (or just transitive) and the point $x_{0}$ is a transitive point.

If $X$ in addition is compact then the pair $\left(X, x_{0}\right)$ is said to be a pointed system or a $G$-ambit. If every point $x$ in a compact $G$-space $X$ is transitive then $X$ is said to be minimal.

Let $G$ act on $X_{1}$ and on $X_{2}$. A continuous map $f: X_{1} \rightarrow X_{2}$ is a $G$-map (or a homomorphism of dynamical systems) if $f(g x)=g f(x)$ for every $(g, x) \in G \times X_{1}$.

A right action $X \times G \rightarrow X$ can be defined analogously. If $G^{\text {op }}$ is the opposite group of $G$ with the same topology then the right $G$-space $(X, G)$ can be treated as a left $G^{\text {op }}$-space $\left(G^{\text {op }}, X\right)$ (and vice versa). A map $h: G_{1} \rightarrow G_{2}$ between two groups is a co-homomorphism (or an anti-homomorphism) if $h\left(g_{1} g_{2}\right)=h\left(g_{2}\right) h\left(g_{1}\right)$. This happens iff $h: G_{1}^{\text {op }} \rightarrow G_{2}$ (the same assignment) is a homomorphism.

The Banach algebra (under the supremum norm) of all continuous realvalued bounded functions on a topological space $X$ will be denoted by 
$C(X)$. Let $(G, X)$ be a left (not necessarily compact) $G$-space. Then it induces the right action $C(X) \times G \rightarrow C(X)$, with $(f g)(x)=f(g x)$, and the corresponding co-homomorphism $h: G \rightarrow \operatorname{Iso}(C(X))$. While the $g$ translations $C(X) \rightarrow C(X)$ (being isometric) are continuous, the orbit maps $\tilde{f}: G \rightarrow C(X), g \mapsto f g$, are not necessarily continuous. The function $f \in C(X)$ is right uniformly continuous if the orbit map $G \rightarrow C(X), g \mapsto f g$, is norm continuous. The set $R U C(X)$ of all right uniformly continuous functions on $X$ is a uniformly closed $G$-invariant subalgebra of $C(X)$. Here and in the following, "subalgebra" means a uniformly closed unital (containing the constants) subalgebra. A $G$-subalgebra is an algebra which is invariant under the natural right action of $G$.

Every topological group $G$ can be treated as a $G$-space under the left regular action of $G$ on itself. In this particular case $f \in R U C(G)$ iff $f$ is uniformly continuous with respect to the right uniform structure $\mathcal{R}$ on $G$ (furthermore, this is also true for coset $G$-spaces $G / H$ ).

Thus, $f \in R U C(G)$ iff for every $\varepsilon>0$ there exists a neighborhood $V$ of the identity element $e \in G$ such that $\sup _{g \in G}|f(v g)-f(g)|<\varepsilon$ for every $v \in V$.

Analogously one defines right translations $(g f)(x):=f(x g)$, and the algebra $L U C(G)$ of left uniformly continuous functions. These are the functions which are uniformly continuous with respect to the left uniform structure $\mathcal{L}$ on $G$.

A $G$-compactification of a $G$-space $X$ is a $G$-map $\nu: X \rightarrow Y$ into a compact $G$-space $Y$ with $\operatorname{cl} \nu(X)=Y$. A compactification is proper when $\nu$ is a topological embedding. Given a compact $G$-space $X$ and a point $x_{0} \in X$ the map $\nu: G \rightarrow X$ defined by $\nu(g)=g x_{0}$ is a compactification of the $G$-space $G$ (the left regular action) in the orbit closure $\operatorname{cl} G x_{0} \subset X$.

We say that a $G$-compactification $\nu: G \rightarrow S$ of $X:=G$ is a right topological semigroup compactification of $G$ if $S$ is a right topological semigroup (that is, $S$ is a compact semigroup such that for every $p \in S$ the map $S \rightarrow S$, $s \mapsto s p$, is continuous) and $\nu$ is a homomorphism of semigroups.

There exists a canonical 1-1 correspondence between the $G$-compactifications of $X$ and $G$-subalgebras of $R U C(X)$ (see for example [50]). The compactification $\nu: X \rightarrow Y$ induces an isometric $G$-embedding of $G$-algebras

$$
j_{\nu}: C(Y)=R U C(Y) \hookrightarrow R U C(X), \quad \phi \mapsto \phi \circ \nu,
$$

and the algebra $\mathcal{A}_{\nu}$ is defined as the image $j_{\nu}(C(Y))$. Conversely, if $\mathcal{A}$ is a $G$-subalgebra of $R U C(X)$, then denote by $X^{\mathcal{A}}$ or by $|\mathcal{A}|$ the corresponding Gelfand space treating it as a weak* compact subset of the dual space $\mathcal{A}^{*}$. It has a structure of a $G$-space $(G,|\mathcal{A}|)$ and the natural map $\nu_{\mathcal{A}}: X \rightarrow X^{\mathcal{A}}, x \mapsto \operatorname{eva}_{x}$, where $\operatorname{eva}_{x}(\varphi):=\varphi(x)$, is the evaluation at $x$ (a multiplicative functional), defines a $G$-compactification. If $\nu_{1}: X \rightarrow X^{\mathcal{A}_{1}}$ 
and $\nu_{2}: X \rightarrow X^{\mathcal{A}_{2}}$ are two $G$-compactifications then $\mathcal{A}_{\nu_{1}} \subset \mathcal{A}_{\nu_{2}}$ iff $\nu_{1}=\alpha \circ \nu_{2}$ for some $G$-map $\alpha: X^{\mathcal{A}_{2}} \rightarrow X^{\mathcal{A}_{1}}$. The algebra $\mathcal{A}$ determines the compactification $\nu_{\mathcal{A}}$ uniquely, up to the equivalence of $G$-compactifications. The $G$ algebra $R U C(X)$ defines the corresponding Gelfand space $|R U C(X)|$, which we denote by $\beta_{G} X$, and the maximal $G$-compactification $i_{\beta}: X \rightarrow \beta_{G} X$. Note that this map may not be an embedding even for Polish $G$ and $X$ (see [24]); it follows that there is no proper $G$-compactification for such $X$. If $X$ is a compact $G$-space then $\beta_{G} X$ can be identified with $X$ and $C(X)=R U C(X)$.

Denote by $G^{R U C}$ the Gelfand space of the $G$-algebra $R U C(G)$. The canonical embedding $u: G \rightarrow G^{R U C}$ defines the greatest ambit $\left(G^{R U C}, u(e)\right)$ of $G$.

It is easy to see that the intersection $U C(G):=R U C(G) \cap L U C(G)$ is a left and right $G$-invariant closed subalgebra of $R U C(G)$. We denote the corresponding compactification by $G^{U C}$. Denote by $\mathcal{L} \wedge \mathcal{R}$ the lower uniformity of $G$. It is the infimum (greatest lower bound) of the left and right uniformities on $G$; we call it the Roelcke uniformity. Clearly, for every bounded function $f: G \rightarrow \mathbb{R}$ we have $f \in U C(G)$ iff $f:(G, \mathcal{L} \wedge \mathcal{R}) \rightarrow \mathbb{R}$ is uniformly continuous. Recall the following important fact (in general the infimum $\mu_{1} \wedge \mu_{2}$ of two compatible uniform structures on a topological space $X$ need not be compatible with the topology of $X$ ).

LEMMA 2.1.

(1) (Roelcke-Dierolf [41]) For every topological group $G$ the Roelcke uniform structure $\mathcal{L} \wedge \mathcal{R}$ generates the given topology of $G$.

(2) For every topological group $G$ the algebra $U C(G)$ separates points from closed subsets in $G$.

Proof. (1) See Roelcke-Dierolf [41, Proposition 2.5].

(2) Follows from (1).

By a uniform $G$-space $(X, \mu)$ we mean a $G$-space $(X, \tau)$ where $\tau$ is the (completely regular) topology defined by the uniform structure $\mu$ and the $g$-translations $(g \in G)$ are uniform isomorphisms.

Let $X:=(X, \mu)$ be a uniform $G$-space. A point $x_{0} \in X$ is a point of equicontinuity (notation: $x_{0} \in \mathrm{Eq}_{X}$ ) if for every entourage $\varepsilon \in \mu$, there is a neighborhood $O$ of $x_{0}$ such that $\left(g x_{0}, g x\right) \in \varepsilon$ for every $x \in O$ and $g \in G$. The $G$-space $X$ is equicontinuous if $\mathrm{Eq}_{X}=X$. The space $(X, \mu)$ is uniformly equicontinuous if for every $\varepsilon \in \mu$ there is $\delta \in \mu$ such that $(g x, g y) \in \varepsilon$ for every $g \in G$ and $(x, y) \in \delta$. For compact $X$ (equipped with the unique compatible uniformity), equicontinuity and uniform equicontinuity coincide. Compact (uniformly) equicontinuous $G$-space $X$ is also said to be almost periodic (for short: $\mathrm{AP}$ ); see also Section 7 . If $\mathrm{Eq}_{X}$ is dense in $X$ then $(X, \mu)$ is said to be an almost equicontinuous (AE) $G$-space [2]. 
The following definition is standard (for more details see for example [13]).

DEFINITION 2.2 .

(1) A function $f \in C(X)$ on a $G$-space $X$ comes from a compact $G$ system $Y$ if there exist a $G$-compactification $\nu: X \rightarrow Y$ (so $\nu$ is onto if $X$ is compact) and a function $F \in C(Y)$ such that $f=F \circ \nu$ (i.e., if $\left.f \in \mathcal{A}_{\nu}\right)$. Then necessarily $f \in R U C(X)$.

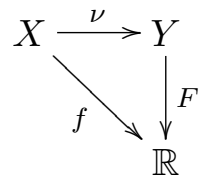

(2) A function $f \in R U C(G)$ comes from a pointed system $\left(Y, y_{0}\right)$ if for some continuous function $F \in C(Y)$ we have $f(g)=F\left(g y_{0}\right)$ for all $g \in G$. Notation: $f \in \mathcal{A}\left(Y, y_{0}\right)$. Defining $\nu: X=G \rightarrow Y$ by $\nu(g)=g y_{0}$ observe that this is indeed a particular case of $2.2(1)$.

(3) Let $\Gamma$ be a class of compact $G$-spaces. For a $G$-space $X$ denote by $\Gamma(X)$ the class of all functions on $X$ which come from a $G$ compactification $\nu: X \rightarrow Y$ where the $G$-system $Y$ belongs to $\Gamma$.

Let $\mathrm{P}$ be a class of compact $G$-spaces which is preserved by $G$-isomorphisms, products and closed $G$-subspaces. It is well known (see for example [13, Proposition 2.9]) that for every $G$-space $X$ there exists a universal (maximal) $G$-compactification $X \rightarrow X^{\mathcal{P}}$ such that $X^{\mathcal{P}}$ lies in P. More precisely, for every (not necessarily compact) $G$-space $X$ denote by $\mathcal{P} \subset C(X)$ the collection of functions coming from $G$-spaces having property P. Then $\mathcal{P}$ is a uniformly closed, $G$-invariant subalgebra of $R U C(X)$ and the maximal $G$-compactification of $X$ with property $\mathrm{P}$ is the corresponding Gelfand space $X^{\mathcal{P}}:=|\mathcal{P}|$. If $X$ is compact then $\left(G, X^{\mathcal{P}}\right)$ is the maximum factor of $(G, X)$ with property $\mathrm{P}$. In particular let $\mathrm{P}$ be one of the following natural classes of compact $G$-spaces: a) almost periodic (= equicontinuous); b) weakly almost periodic; c) hereditarily nonsensitive; d) locally equicontinuous; e) all compact $G$-spaces. Then, in this way the following maximal (in the corresponding class) $G$-compactifications are: a) $G^{A P}$; b) $G^{W A P}$; c) $G^{\text {Asp }}$; d) $G^{L E}$; e) $G^{R U C}$.

For undefined concepts and more details see Section 7 and also [13].

3. Cyclic $G$-systems and point-universality. Here we give some background material about cyclic compact $G$-systems $X_{f}$ defined for $f \in$ $R U C(X)$. These $G$-spaces play a significant role in many aspects of topological dynamics and are well known at least for the particular case of $X:=G$. We mostly use the presentation and results of [13] (see also [51]). 
As a first motivation note a simple fact about Definition 2.2. For every $G$-space $X$ a function $f: X \rightarrow \mathbb{R}$ lies in $R U C(X)$ iff it comes from a compact $G$-flow $Y$. We can choose $Y$ via the maximal $G$-compactification $G \rightarrow \beta_{G} G=Y$ of $G$. This is the largest possibility in this setting. Among all possible $G$-compactifications $\nu: X \rightarrow Y$ of $X$ such that $f$ comes from $(\nu, Y)$ there exists also the smallest one. Take simply the smallest $G$-subalgebra $\mathcal{A}_{f}$ of $R U C(X)$ generated by the orbit $f G$ of $f$ in $R U C(X)$. Denote by $X_{f}$ the Gelfand space $\left|\mathcal{A}_{f}\right|=X^{\mathcal{A}_{f}}$ of the algebra $\mathcal{A}_{f}$. Then the corresponding $G$-compactification $X \rightarrow Y:=X_{f}$ is the desired one. We call $\mathcal{A}_{f}$ and $X_{f}$ the cyclic $G$-algebra and cyclic $G$-system of $f$, respectively. Next we provide an alternative construction and some basic properties of $X_{f}$.

Let $X$ be a (not necessarily compact) $G$-space. Given $f \in R U C(X)$ let $I=[-\|f\|,\|f\|] \subset \mathbb{R}$ and $\Omega=I^{G}$, the product space equipped with the compact product topology. We let $G$ act on $\Omega$ by $g \omega(h)=\omega(h g), g, h \in G$. Define the continuous map

$$
f_{\sharp}: X \rightarrow \Omega, \quad f_{\sharp}(x)(g)=f(g x),
$$

and the closure $X_{f}:=\operatorname{cl}\left(f_{\sharp}(X)\right)$ in $\Omega$. Note that $X_{f}=f_{\sharp}(X)$ whenever $X$ is compact.

Denoting the unique continuous extension of $f$ to $\beta_{G} X$ by $\tilde{f}$ (it exists because $f \in R U C(X))$ we now define a map

$$
\psi: \beta_{G} X \rightarrow X_{f} \quad \text { by } \quad \psi(y)(g)=\widetilde{f}(g y), \quad y \in \beta_{G} X, g \in G .
$$

Let $\operatorname{pr}_{e}: \Omega \rightarrow \mathbb{R}$ denote the projection of $\Omega=I^{G}$ onto the $e$-coordinate and let $F_{e}:=\operatorname{pr}_{e} \uparrow_{X_{f}}: X_{f} \rightarrow \mathbb{R}$ be its restriction to $X_{f}$. Thus, $F_{e}(\omega):=\omega(e)$ for every $\omega \in X_{f}$.

As before denote by $\mathcal{A}_{f}$ the smallest (closed and unital, of course) $G$ invariant subalgebra of $R U C(X)$ which contains $f$. There is then a naturally defined $G$-action on the Gelfand space $X^{\mathcal{A}_{f}}=\left|\mathcal{A}_{f}\right|$ and a $G$-compactification (morphism of dynamical systems if $X$ is compact) $\pi_{f}: X \rightarrow\left|\mathcal{A}_{f}\right|$. Next consider the map $\pi: \beta_{G} X \rightarrow\left|\mathcal{A}_{f}\right|$, the canonical extension of $\pi_{f}$ induced by the inclusion $\mathcal{A}_{f} \subset R U C(X)$.

The action of $G$ on $\Omega$ is not in general continuous. However, the restricted action on $X_{f}$ is continuous for every $f \in R U C(X)$. This follows from the second assertion of the next fact.

Proposition 3.1 (see for example [13]).

(1) Each $\omega \in X_{f}$ is an element of $R U C(G)$. That is, $X_{f} \subset R U C(G)$.

(2) The map $\psi: \beta_{G} X \rightarrow X_{f}$ is a continuous homomorphism of $G$ systems. The dynamical system $\left(G,\left|\mathcal{A}_{f}\right|\right)$ is isomorphic to $\left(G, X_{f}\right)$ and the diagram 


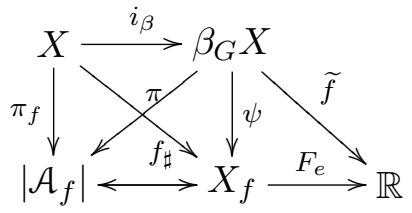

commutes.

(3) $f=F_{e} \circ f_{\sharp}$. Thus every $f \in R U C(X)$ comes from the system $X_{f}$. Moreover, if $f$ comes from a system $Y$ and a $G$-compactification $\nu: X \rightarrow Y$ then there exists a homomorphism $\alpha: Y \rightarrow X_{f}$ such that $f_{\sharp}=\alpha \circ \nu$. In particular, $f \in \mathcal{A}_{f} \subset \mathcal{A}_{\nu}$.

If $X:=G$ with the usual left action then $X_{f}$ is the pointwise closure of the $G$-orbit $G f:=\{g f\}_{g \in G}$ of $f$ in $R U C(G)$. Hence $\left(X_{f}, f\right)$ is a transitive pointed $G$-system.

As expected by the construction the cyclic $G$-systems $X_{f}$ provide "building blocks" for compact $G$-spaces. That is, every compact $G$-space can be embedded into the $G$-product of $G$-spaces $X_{f}$.

Let us say that a topological group $G$ is uniformly Lindelöf if for every nonempty open subset $O \subset G$ countably many translates $g_{n} O$ cover $G$ (there are several alternative names for this notion: $\omega$-bounded, $\omega$-bounded, $\omega$-narrow, $\omega$-precompact). It is well known that $G$ is uniformly Lindelöf iff $G$ is a topological subgroup in a product of second countable groups. When $G$ is uniformly Lindelöf (e.g. when $G$ is second countable) the compactum $X_{f}$ is metrizable.

The question "when is $X_{f}$ a subset of $U C(G)$ ?" provides another motivation for introducing the notion of strongly uniformly continuous (SUC) functions (see Definition 4.2 and Theorem 4.12).

The enveloping (or Ellis) semigroup $E=E(X)$ of a compact $G$-space $X$ is defined as the closure in $X^{X}$ (with its compact pointwise convergence topology) of the set $\left\{\pi^{g}: X \rightarrow X\right\}_{g \in G}$ of translations considered as a subset of $X^{X}$. With the operation of composition of maps this is a right topological semigroup. Moreover, the map

$$
j=j_{X}: G \rightarrow E(X), \quad g \mapsto \pi^{g},
$$

is a right topological semigroup compactification of $G$. The compact space $E(X)$ becomes a $G$-space with respect to the natural action

$$
G \times E(X) \rightarrow E(X), \quad(g p)(x)=g p(x) .
$$

Moreover, the pointed $G$-system $(E(X), j(e))$ is point-universal in the following sense.

Definition 3.2 ([13]). A pointed $G$-system $\left(X, x_{0}\right)$ is point-universal if it has the property that for every $x \in X$ there is a homomorphism $\pi_{x}$ : 
$\left(X, x_{0}\right) \rightarrow(\operatorname{cl}(G x), x)$. The $G$-subalgebra $\mathcal{A} \subset R U C(G)$ is said to be pointuniversal if the corresponding $G$-ambit $\left(G^{\mathcal{A}}, u_{\mathcal{A}}(e)\right)$ is point-universal.

We will use the following characterization of point-universality from [13].

Lemma 3.3. Let $\left(X, x_{0}\right)$ be a transitive compact $G$-system. The following conditions are equivalent:

(1) The system $\left(X, x_{0}\right)$ is point-universal.

(2) The orbit map $G \rightarrow X, g \mapsto g x_{0}$, is a right topological semigroup compactification of $G$.

(3) $\left(X, x_{0}\right)$ is $G$-isomorphic to its enveloping semigroup $(E(X), j(e))$.

(4) $\mathcal{A}\left(X, x_{0}\right)=\bigcup_{x \in X} \mathcal{A}(\operatorname{cl}(G x), x)$.

(5) $X_{f} \subset \mathcal{A}\left(X, x_{0}\right)$ for every $f \in \mathcal{A}\left(X, x_{0}\right)$ (where $\mathcal{A}\left(X, x_{0}\right)$ is the corresponding subalgebra of $R U C(G)$ coming from the $G$-compactification $\left.\nu: G \rightarrow X, \nu(g)=g x_{0}\right)$.

In particular, for every right topological semigroup compactification $\nu$ : $G \rightarrow S$ the pointed $G$-space $(S, \nu(e))$ is point-universal. For other properties of point-universality see [13] and Remark 9.4.

4. Strong uniform continuity. Let $G$ be a topological group. As before denote by $\mathcal{L}$ and $\mathcal{R}$ the left and right uniformities on $G$. We start with a simple observation.

Lemma 4.1. For every compact $G$-space $X$ the corresponding orbit maps

$$
\widetilde{x}:(G, \mathcal{R}) \rightarrow\left(X, \mu_{X}\right), \quad g \mapsto g x,
$$

are uniformly continuous for every $x \in X$.

Proof. Let $V$ be an open neighborhood of the diagonal $\Delta \subset X \times X$. In order to obtain a contradiction suppose that for every neighborhood $U$ of $e \in G$ there are $u_{U} \in U$ and $x_{U} \in X$ such that $\left(x_{U}, u_{U} x_{U}\right) \notin V$. For a convergent subnet we have $\lim \left(x_{U}, u_{U} x_{U}\right)=\left(x, x^{\prime}\right) \notin V$, contradicting the joint continuity of the $G$-action.

In general, for noncommutative groups, one cannot replace $\mathcal{R}$ by the left uniformity $\mathcal{L}$ (see Remark 4.4). This leads us to the following definition.

Definition 4.2. Let $G$ be a topological group.

(1) We say that a uniform $G$-space $(X, \mu)$ is strongly uniformly continuous at $x_{0} \in X$ (notation: $x_{0} \in S U C_{X}$ ) if the orbit map $\widetilde{x}_{0}: G \rightarrow X$, $g \mapsto g x_{0}$, is $(\mathcal{L}, \mu)$-uniformly continuous. Precisely, this means that for every $\varepsilon \in \mu$ there exists a neighborhood $U$ of $e \in G$ such that

$$
\left(\text { gux }_{0}, g x_{0}\right) \in \varepsilon
$$

for every $g \in G$ and every $u \in U$. If $S U C_{X}=X$ we say that $X$ is strongly uniformly continuous. 
(2) If $X$ is a compact $G$-space then there exists a unique compatible uniformity $\mu_{X}$ on $X$. So, $S U C_{X}$ is well defined. By Lemma 4.1 it follows that a compact $G$-space $X$ is SUC at $x_{0}$ iff $\widetilde{x}_{0}: G \rightarrow X$ is $\left(\mathcal{L} \wedge \mathcal{R}, \mu_{X}\right)$-uniformly continuous. We let SUC denote the class of all compact $G$-systems such that $\left(X, \mu_{X}\right)$ is SUC.

(3) A function $f \in C(X)$ is strongly uniformly continuous (notation: $f \in S U C(X))$ if it comes from a SUC compact dynamical system.

(4) Let $x_{1}$ and $x_{2}$ be points of a $G$-space $X$. Write $x_{1} \stackrel{S U C}{\sim} x_{2}$ if these points cannot be separated by a SUC function on $X$. Equivalently, this means that these points have the same images under the universal SUC compactification $G$-map $X \rightarrow X^{S U C}$ (see Lemma 4.6(1) below).

Lemma 4.3. $S U C(G) \subset U C(G)$.

Proof. Let $f: G \rightarrow \mathbb{R}$ belong to $S U C(G)$. Then it comes from a function $F: X \rightarrow \mathbb{R}$, where $\nu: G \rightarrow X$ is a $G$-compactification of $G$ such that $f(g)=F(\nu(g))$, and $F \in S U C(X)$. Clearly, $F$ is uniformly continuous because $X$ is compact. Then $f \in R U C(G)$ by Lemma 4.1. In order to see that $f \in L U C(G)$, choose $x_{0}:=\nu(e) \in X$ in the definition of SUC.

REMARK 4.4. Recall that if $\mathcal{L}=\mathcal{R}$ then $G$ is said to be a SIN group. If $G$ is a SIN group then $X \in \mathrm{SUC}$ for every compact $G$-space $X$. It follows that for a SIN group $G$ we have $S U C(X)=R U C(X)$ for every, not necessarily compact, $G$-space $X$ and also $S U C(G)=U C(G)=R U C(G)$. For example this holds for abelian, discrete, and compact groups.

A special case of a SUC uniform $G$-space is obtained when the uniform structure $\mu$ is defined by a $G$-invariant metric. If $\mu$ is a metrizable uniformity then $(X, \mu)$ is uniformly equicontinuous iff $\mu$ can be generated by a $G$-invariant metric on $X$. A slightly sharper property is the local version: $S U C_{X} \supset \mathrm{Eq}_{X}$ (see Lemma 7.8).

We say that a compactification of $G$ is Roelcke if the corresponding algebra $\mathcal{A}$ is a $G$-subalgebra of $U C(G)$, or equivalently, if there exists a natural $G$-morphism $G^{U C} \rightarrow G^{\mathcal{A}}$.

LEMMA 4.5.

(1) Let $f:(X, \mu) \rightarrow(Y, \eta)$ be a uniformly continuous $G$-map. Then $f\left(S U C_{X}\right) \subset S U C_{Y}$.

(2) The class $S U C$ is closed under products, subsystems and quotients.

(3) Let $\alpha: G \rightarrow Y$ be a Roelcke compactification. Then $\alpha(G) \subset S U C_{Y}$.

(4) Let $X$ be a not necessarily compact $G$-space and $f \in S U C(X)$. Then for every $x_{0} \in X$ and every $\varepsilon>0$ there exists a neighborhood $U$ of $e$ 
such that

$$
\left|f\left(g u x_{0}\right)-f\left(g x_{0}\right)\right|<\varepsilon \quad \forall(g, u) \in G \times U .
$$

Proof. (1) and (2) are straightforward.

(3): Follows directly from (1) because the left action of $G$ on itself is uniformly equicontinuous with respect to the left uniformity and $\alpha:(G, \mathcal{L}) \rightarrow$ $\left(Y, \mu_{Y}\right)$ is uniformly continuous for every Roelcke compactification.

(4): There exist: a compact SUC $G$-space $Y$, a continuous function $F$ : $Y \rightarrow \mathbb{R}$ and a $G$-compactification $\nu: X \rightarrow Y$ such that $f=F \circ \nu$. Now our assertion follows from the fact that $\nu\left(x_{0}\right) \in S U C_{Y}$ for every $x_{0} \in X$ (taking into account that $F$ is uniformly continuous).

By Lemmas 4.5(2), 3.3 and the standard subdirect product construction (see [13, Proposition 2.9(2)]) we can derive the following facts.

LEMMA 4.6.

(1) The collection $S U C(X)$ is a $G$-subalgebra of $R U C(X)$ for every $G$ space $X$, and the corresponding Gelfand space $|S U C(X)|=X^{S U C}$ with the canonical compactification $j: X \rightarrow X^{S U C}$ is the maximal $S U C$-compactification of $X$.

(2) A compact $G$-system $X$ is $S U C$ if and only if $C(X)=S U C(X)$.

(3) For every $f \in R U C(X)$ we have $f \in S U C(X)$ if and only if $X_{f}$ is $S U C$.

(4) $\operatorname{SUC}(G)$ is a point-universal closed $G$-subalgebra of $R U C(G)$.

(5) The canonical compactification $j: G \rightarrow G^{S U C}$ is always a right topological semigroup compactification of $G$.

We also need the following link between SUC functions and cyclic $G$ spaces.

Lemma 4.7. Let $X$ be a $G$-space, $f \in R U C(X)$ and $h \in X_{f}$. Then the following are equivalent:

(1) $h \in L U C(G)$.

(2) $h \in U C(G)$.

(3) $h$, as a point in the $G$-flow $Y:=X_{f}$, is in $S U C_{Y}$.

Proof. We know by Proposition 3.1(1) that $X_{f} \subset R U C(G)$. Thus (1) $\Leftrightarrow(2)$. For $(1) \Leftrightarrow(3)$ observe that $|h(g u)-h(g)|<\varepsilon \quad \forall g \in G \Leftrightarrow\left|h\left(t_{k} g u\right)-h\left(t_{k} g\right)\right|<\varepsilon \quad \forall g \in G, k=1, \ldots, n$ for arbitrary finite subset $\left\{t_{1}, \ldots, t_{n}\right\}$ of $G$.

The following result shows that $S U C(X)$ can be described by internal terms for every compact $G$-space $X$. 
Proposition 4.8. Let $X$ be a compact $G$-space. The following are equivalent:

(1) $f \in S U C(X)$.

(2) For every $x_{0} \in X$ and every $\varepsilon>0$ there exists a neighborhood $U$ of $e$ such that

$$
\left|f\left(g u x_{0}\right)-f\left(g x_{0}\right)\right|<\varepsilon \quad \forall(g, u) \in G \times U .
$$

Proof. (1) $\Rightarrow(2)$ : Apply Lemma 4.5(4).

$(1) \Leftarrow(2)$ : By Lemma $4.6(3)$ we have to show that the cyclic $G$-space $X_{f}$ is SUC. Fix an arbitrary element $\omega \in X_{f}$. According to Lemma 4.7 it is equivalent to verify that $\omega \in L U C(G)$. The $G$-compactification map $f_{\sharp}: X \rightarrow X_{f}$ is onto because $X$ is compact. Choose $x_{0} \in X$ such that $f_{\sharp}\left(x_{0}\right)=\omega$. Then $\omega(g)=f\left(g x_{0}\right)$ for every $g \in G$. By assertion (2) for $x_{0} \in X$ and $\varepsilon$ we can pick a neighborhood $U$ of $e$ such that

$$
\left|f\left(g u x_{0}\right)-f\left(g x_{0}\right)\right| \leq \varepsilon \quad \forall(g, u) \in G \times U .
$$

Now we can finish the proof by observing that

$$
\left|f\left(g u x_{0}\right)-f\left(g x_{0}\right)\right|=|\omega(g u)-\omega(g)| \leq \varepsilon \quad \forall(g, u) \in G \times U .
$$

The following result emphasizes the differences between $R U C(G)$ and $\operatorname{SUC}(G)$.

THEOREM 4.9. Let $\alpha=\alpha_{\mathcal{A}}: G \rightarrow S$ be a $G$-compactification of $G$ such that the corresponding left $G$-invariant subalgebra $\mathcal{A}$ of $R U C(G)$ is also right $G$-invariant. Consider the following conditions:

(1) $\mathcal{A} \subset U C(G)$ (that is, $\alpha: G \rightarrow S$ is a Roelcke compactification).

(2) The induced right action $S \times G \rightarrow S,(s, g) \mapsto s \alpha(g)$, is jointly continuous.

(3) $\mathcal{A} \subset S U C(G)$.

Then

(a) Always, (1) $\Leftrightarrow(2)$ and $(3) \Rightarrow(1)$.

(b) If , in addition, $S$ is a right topological semigroup and $\alpha: G \rightarrow S$ is a right topological semigroup compactification of $G$ then $(1) \Leftrightarrow(2) \Leftrightarrow(3)$.

Proof. (a) $(1) \Leftrightarrow(2)$ : By our assumption, $\mathcal{A}$ is $G$-invariant with respect to left and right translations (that is, the functions $(f g)(x):=f(g x)$ and $(g f)(x):=f(x g)$ lie in $\mathcal{A}$ for every $f \in \mathcal{A}$ and $g \in G)$. Then the corresponding (weak* compact) Gelfand space $S:=X^{\mathcal{A}} \subset \mathcal{A}^{*}$ admits the natural dual left and right actions (see also Definition 6.2 and Remark 6.7) $\pi_{l}: G \times S \rightarrow S$ and $\pi_{r}: S \times G \rightarrow S$ such that $\left(g_{1} s\right) g_{2}=g_{1}\left(s g_{2}\right)$ for every $\left(g_{1}, s, g_{2}\right) \in G \times S \times G$. It is easy to see that this right action $S \times G \rightarrow S$ is jointly continuous if and only if $\mathcal{A} \subset L U C(G)$. On the other 
hand, since $\alpha: G \rightarrow S$ is a $G$-compactification of left $G$-spaces we already have $\mathcal{A} \subset R U C(G)$.

$(3) \Rightarrow(1)$ : By Lemma 4.3 we have $S U C(G) \subset U C(G)$.

(b) We have to verify that $(1) \Rightarrow(3)$ provided that $\alpha: G \rightarrow S$ is a right topological semigroup compactification of $G$. The latter condition is equivalent to the fact that the system $(G, S)$ is point-universal (Lemma 3.3) and thus for every $x_{0} \in S$ there exists a homomorphism of $G$-ambits $\phi:(S, \alpha(e)) \rightarrow\left(\operatorname{cl}\left(G x_{0}\right), x_{0}\right)$. By Lemma 4.5 we conclude that the point $x_{0}=\phi(\alpha(e)) \in S$ is a point of SUC in the $G$-system $\operatorname{cl}\left(G x_{0}\right)$ (and hence in $S$ ). Since $x_{0}$ is an arbitrary point in $S$ we see that $S U C_{S}=S$ and hence $S$ is an SUC system. Since every function $f \in \mathcal{A}$ on $G$ comes from the compactification $\alpha: G \rightarrow S$ we conclude that $\mathcal{A} \subset S U C(G)$.

COROLlary 4.10. The G-compactification $j: G \rightarrow G^{S U C}$ is a right topological semigroup compactification of $G$ such that the right action $G^{S U C}$ $\times G \rightarrow G^{S U C}$ is also jointly continuous.

Proof. Apply Proposition 4.6(5) and Theorem 4.9.

COROLlary 4.11. The following conditions are equivalent:

(1) $i: G \rightarrow G^{U C}$ is a right topological semigroup compactification.

(2) $\left(G^{U C}, i(e)\right)$ is a point-universal $G$-system.

(3) $G^{U C}$ is $S U C$.

(4) $\operatorname{SUC}(G)=U C(G)$.

Proof. Apply assertion (b) of Theorem 4.9 to $\mathcal{A}=U C(G)$ taking into account Lemmas 3.3 and 4.3.

Particularly interesting examples of groups $G$ with $S U C(G)=U C(G)$ are the Polish groups $U(H)$ of all unitary operators (Example 7.13), and the group $S_{\infty}(\mathbb{N})$ (Theorem 12.2). In both cases we actually have $\operatorname{SUC}(G)=$ $U C(G)=W A P(G)$. Note that these groups are not SIN (cf. Remark 4.4).

For the next result see also Veech [49, Section 5].

TheOREM 4.12. Let $f \in R U C(X)$. The following conditions are equivalent:

(1) $X_{f} \subset U C(G)$.

(2) $\left(G, X_{f}\right)$ is $S U C$.

(3) $f \in S U C(X)$.

Proof. (1) $\Rightarrow(2)$ : Let $h \in X_{f}$. By our assumption we have $h \in U C(G) \subset$ $L U C(G)$. Then by Lemma $4.7, h$, as a point in the $G$-flow $Y:=X_{f}$, is in $S U C_{Y}$. So $S U C_{Y}=Y$. This means that $\left(G, X_{f}\right)$ is SUC.

$(2) \Rightarrow(3)$ : Let $\left(G, X_{f}\right)$ be SUC. By Proposition 3.1.3, the function $f$ : $X \rightarrow \mathbb{R}$ comes from the $G$-compactification $f_{\sharp}: X \rightarrow X_{f}$. By Definition 4.2.3 this means that $f \in S U C(X)$. 
$(3) \Rightarrow(1)$ : Let $f \in S U C(X)$. Then Lemma 4.6.3 says that $X_{f}$ is SUC. By Lemma 4.7 we have $X_{f} \subset U C(G)$.

5. SUC, homogeneity and the epimorphism problem. We say that a $G$-space $X$ is a coset $G$-space if it is $G$-isomorphic to the usual coset $G$-space $G / H$ where $H$ is a closed subgroup of $G$ and $G / H$ is equipped with the quotient topology. We say that a $G$-space $X$ is homogeneous if for every $x, y \in X$ there exists $g \in G$ such that $g x=y$. A homogeneous $G$-space $X$ is a coset $G$-space if and only if the orbit map $\widetilde{x}: G \rightarrow X$ is open for some (equivalently, every) $x \in X$. Furthermore, $\widetilde{x}: G \rightarrow X$ is open iff it is a quotient map. Recall that by a well known result of Effros every homogeneous $G$-space with Polish $G$ and $X$ is necessarily a coset $G$-space.

Proposition 5.1. Let $X=G / H$ be a compact coset $G$-space.

(1) If $X$ is SUC then $X$ is equicontinuous (that is, almost periodic).

(2) $S U C(X)=A P(X)$.

Proof. (1): Indeed, let $x_{0} H \in G / H$ and let $\varepsilon$ be an element of the uniform structure on the compact space $X$. By Definition 4.2 we can choose a neighborhood $U$ of $e$ such that

$$
\left(g u x_{0} H, g x_{0} H\right) \in \varepsilon \quad \forall(g, u) \in G \times U .
$$

By the definition of coset space topology the set $O:=U x_{0} H$ is a neighborhood of the point $x_{0} H$ in $G / H$. We see that $\left(g x H, g x_{0} H\right) \in \varepsilon$ whenever $x H \in O$. This proves that $x_{0} H$ is a point of equicontinuity of $X=G / H$. Hence $X$ is AP.

(2): Every equicontinuous compact $G$-space is clearly SUC. This implies that always $S U C(X) \supset A P(X)$. Conversely, let $f \in S U C(X)$. This means that $f=\alpha \circ F$ for a $G$-compactification $\alpha: X \rightarrow Y$ where $Y$ is SUC and $F \in C(Y)=S U C(Y)$. We can suppose that $\alpha$ is onto because $X$ is compact. Then $\alpha$ is a quotient map. On the other hand, $X$ is a coset space $G / H$. It follows that the natural onto map $G \rightarrow Y$ is also a quotient map. Therefore, $Y$ is also a coset space of $G$. Now we can apply (1). It follows that $Y$ is almost periodic. Hence $f$ comes from an AP $G$-factor $Y$ of $X$. Thus, $f \in A P(X)$.

Next we discuss a somewhat unexpected connection between SUC, free topological $G$-groups and an epimorphism problem. Uspenskij has shown in [44] that in the category of Hausdorff topological groups, epimorphisms need not have a dense range. This answers a longstanding problem by K. Hofmann. Pestov established $[36,38]$ that the question completely depends on the free topological $G$-groups $F_{G}(X)$ of a $G$-space $X$ in the sense of Megrelishvili [25]. More precisely, the inclusion $i: H \hookrightarrow G$ of topological groups is an epimorphism iff the free topological $G$-group $F_{G}(X)$ of the coset $G$-space 
$X:=G / H$ is trivial. Triviality means, "as trivial as possible", isomorphic to the cyclic discrete group.

For a $G$-space $X$ and points $x_{1}, x_{2} \in X$ we write $x_{1} \stackrel{\text { Aut }}{\sim} x_{2}$ if these two points have the same image under the canonical $G$-map $X \rightarrow F_{G}(X)$. If $d$ is a compatible $G$-invariant metric on a $G$-space $X$ then $F_{G}(X)$ coincides with the usual free topological group $F(X)$ of $X$ (see [25, Proposition 3.11]). Therefore, in this case $x_{1} \stackrel{\text { Aut }}{\sim} x_{2}$ iff $x_{1}=x_{2}$.

TheOREM 5.2. Let $H$ be a closed subgroup of $G$.

(1) If $x_{1} \stackrel{\text { Aut }}{\sim} x_{2}$ for $x_{1}, x_{2}$ in the $G$-space $X:=G / H$ then $x_{1} \stackrel{\text { SUC }}{\sim} x_{2}$.

(2) If the inclusion $H \hookrightarrow G$ is an epimorphism then the coset $G$-space $G / H$ is SUC-trivial.

Proof. (1) Supposing the contrary let $f: G / H \rightarrow \mathbb{R}$ be a SUC function which separates $x_{1}:=a_{1} H$ and $x_{2}:=a_{2} H$. Then the $G$-invariant pseudometric $\varrho_{f}$ on $G / H$ defined by $\varrho_{f}(x H, y H):=\sup _{g \in G}|f(g x H)-f(g y H)|$ also separates these points. We show that $\varrho_{f}$ is continuous. Indeed, let $\varepsilon>0$ and $x_{0} H \in G / H$. By virtue of Lemma 4.5(4) we can choose a neighborhood $U$ of $e$ such that

$$
\left|f\left(g u x_{0} H\right)-f\left(g x_{0} H\right)\right|<\varepsilon \quad \forall(g, u) \in G \times U .
$$

By the definition of coset space topology the set $O:=U x_{0} H$ is a neighborhood of the point $x_{0} H$ in $G / H$. We see that $\varrho_{f}\left(x H, x_{0} H\right)<\varepsilon$ whenever $x H \in O$. This proves the continuity of $\varrho_{f}$.

Consider the associated metric space $(Y, d)$ and the canonical distance preserving onto $G$-map $X \rightarrow Y, x \mapsto[x]$. The metric $d$ on $Y$ (defined by $\left.d([x],[y]):=\varrho_{f}(x, y)\right)$ is $G$-invariant. Then $F_{G}(Y)$ and $F(Y)$ are canonically equivalent (see the discussion above). Since $d\left(\left[x_{1}\right],\left[x_{2}\right]\right)>0$ we conclude that $x_{1}$ and $x_{2}$ have different images in $F_{G}(X)$. This contradicts the assumption $x_{1} \stackrel{\text { Aut }}{\sim} x_{2}$.

(2) Assume that $G / H$ is not SUC-trivial. By (1) we know that the free topological $G$-group $F_{G}(G / H)$ of $G / H$ is not trivial. Therefore by the above mentioned result of Pestov [36] we can conclude that the inclusion $H \hookrightarrow G$ is not an epimorphism.

REMARK 5.3.

(1) The converse to Theorem 5.2(2) is not true (take $G:=H_{+}[0,1]$, $H:=\{e\}$ and apply Theorem 8.3).

(2) As a corollary of Theorem 5.2(2) one can get several examples of SUC-trivial (compact) $G$-spaces. For example, by [25] the free topological $G$-group $F_{G}(X)$ of $X:=G / H$ with $G:=H(\mathbb{T}), H:=\operatorname{St}(z)$ (where $z \in \mathbb{T}$ is an arbitrary point of the circle $\mathbb{T}$ ) is trivial. In fact, it 
is easy to see that the same is true for the smaller group $G:=H_{+}(\mathbb{T})$ (and the subgroup $H:=\operatorname{St}(z)$ ) (cf. Proposition 5.1).

(3) It is a well known result by Nummela [35] that if $G$ is a SIN group then the inclusion of a closed proper subgroup $H \hookrightarrow G$ is not an epimorphism. This result easily follows from Theorem 5.2(2). Indeed, if $G$ is SIN then by Remark 4.4 for the coset $G$-space $G / H$ we have $S U C(G / H)=R U C(G / H)$. Hence if $G / H$ is SUC-trivial then necessarily $H=G$ because $R U C(G / H)$ is nontrivial for every closed proper subgroup $H$ of $G$.

6. Representations of groups and $G$-spaces on Banach spaces. For a real normed space $V$ denote by $B_{V}$ its closed unit ball $\{v \in V:\|v\| \leq 1\}$. Denote by $\operatorname{Iso}(V)$ the topological group of all linear surjective isometries $V \rightarrow V$ endowed with the strong operator topology. This is just the topology of pointwise convergence inherited from $V^{V}$. Let $V^{*}$ be the dual Banach space of $V$ and

$$
\langle,\rangle: V \times V^{*} \rightarrow \mathbb{R}, \quad(v, \psi) \mapsto\langle v, \psi\rangle=\psi(v)
$$

the canonical (always continuous) bilinear mapping.

A representation (co-representation) of a topological group $G$ on a normed space $V$ is a homomorphism (resp. co-homomorphism) $h: G \rightarrow$ Iso $(V)$. Sometimes it is more convenient to describe a representation (corepresentation) by the corresponding left (resp. right) linear isometric actions $\pi_{h}: G \times V \rightarrow V,(g, v) \mapsto g v=h(v)(g)$ (resp., $V \times G \rightarrow V,(v, g) \mapsto v g=$ $h(v)(g))$. The (co)representation $h$ is continuous if and only if the action $\pi_{h}$ is continuous.

REMARK 6.1. Many results formulated for co-representations remain true also for representations (and vice versa) taking into account the following simple fact: for every representation (resp. co-representation) $h$ there exists an associated co-representation (resp. representation) $h^{\text {op }}: G \rightarrow$ $\operatorname{Iso}(V), g \mapsto h\left(g^{-1}\right)$.

Definition 6.2. Let $\pi: G \times V \rightarrow V$ be a continuous left action of $G$ on $V$ by linear operators. The adjoint (or dual) right action $\pi^{*}: V^{*} \times$ $G \rightarrow V^{*}$ is defined by $\psi g(v):=\psi(g v)$. The corresponding adjoint (dual) left action is $\pi^{*}: G \times V^{*} \rightarrow V^{*}$, where $g \psi(v):=\psi\left(g^{-1} v\right)$. Similarly, if $\pi: V \times G \rightarrow V$ is a continuous linear right action of $G$ on $V$ (e.g., induced by some co-representation), then the corresponding adjoint (dual) action $\pi^{*}: G \times V^{*} \rightarrow V^{*}$ is defined by $g \psi(v):=\psi(v g)$.

The main question considered in [26] was whether the dual action $\pi^{*}$ of $G$ on $V^{*}$ is jointly continuous with respect to the norm topology on $V^{*}$. When this is the case we say that the action $\pi$ (and also the corresponding 
representation $h: G \rightarrow \operatorname{Iso}(V)$, when $\pi$ is an action by linear isometries) is adjoint continuous. (This name was suggested by V. Uspenskij.)

REMARK 6.3. In general, not every continuous representation is adjoint continuous (see for example [26]). A standard example is the representation of the circle group $G:=\mathbb{T}$ on $V:=C(\mathbb{T})$ by translations. Here the Banach space $V$ is separable but with "bad" geometry. The absence of adjoint continuity may happen even for relatively "good" (for instance, separable Radon-Nikodým) Banach spaces like $V:=l_{1}$. Indeed if we consider the symmetric group $G:=S_{\infty}$, naturally embedded into Iso $(V)$ (endowed with the strong operator topology) as the group of "permutation of coordinates" operators, then the dual action of $G$ on $l_{1}^{*}=l_{\infty}$ is not continuous (see [27]).

It turns out that the situation in that respect is the best possible for the important class $\mathcal{A} s p$ of Asplund Banach spaces. The investigation of this class and the closely related Radon-Nikodym property is among the main themes in Banach space theory. Recall that a Banach space $V$ is an Asplund space if the dual of every separable linear subspace is separable, iff every bounded subset $A$ of the dual $V^{*}$ is (weak*, norm)-fragmented, iff $V^{*}$ has the Radon-Nikodým property. Reflexive spaces and spaces of the type $c_{0}(\Gamma)$ are Asplund. For more details cf. $[6,10]$. For the reader's convenience we also recall the definition of fragmentability.

Definition 6.4 (Jayne and Rogers [23]). Let $(X, \tau)$ be a topological space and $\varrho$ be a metric on the set $X$. Then $X$ is said to be $(\tau, \varrho)$-fragmented if for every nonempty $A \subset X$ and every $\varepsilon>0$ there exists a $\tau$-open subspace $O$ of $X$ such that $O \cap A$ is nonempty and $\varepsilon$-small in $(X, \varrho)$.

Namioka's joint continuity theorem implies that every weakly compact set in a Banach space is norm fragmented. This explains why every reflexive space is Asplund.

We will use the following result.

Theorem 6.5 ([26, Corollary 6.9]). Let $V$ be an Asplund Banach space. If a (not necessarily isometric) linear action $\pi: G \times V \rightarrow V$ is continuous then the dual right action $\pi^{*}: V^{*} \times G \rightarrow V^{*}$ is also continuous.

Certainly, this result remains true for dual left actions $\pi^{*}: G \times V^{*} \rightarrow V^{*}$, where $g \psi(v):=\psi\left(g^{-1} v\right)$, as well as for dual actions defined by a right action $\pi: V \times G \rightarrow V$. The obvious reason is the continuity of the map $G \rightarrow$ $G, g \mapsto g^{-1}$.

The following definition provides a flow version of the group representation definitions discussed above. It differs from the usual notion of $G$ linearization in that here we represent the phase space of the flow as a subset of the dual space $V^{*}$ (with respect to the dual action and weak topology) rather than as a subset of $V$. 
Definition 6.6 ([30]). Let $X$ be a $G$-space. A continuous (proper) representation of $(G, X)$ on a Banach space $V$ is a pair

$$
(h, \alpha): G \times X \rightrightarrows \mathrm{Iso}(V) \times B^{*}
$$

where $h: G \rightarrow \operatorname{Iso}(V)$ is a strongly continuous co-homomorphism and $\alpha$ : $X \rightarrow B^{*}$ is a weak* continuous $G$-map (resp. embedding) with respect to the dual action $G \times V^{*} \rightarrow V^{*},(g \varphi)(v):=\varphi(h(g)(v))$. Here $B^{*}$ is the weak ${ }^{*}$ compact unit ball of the dual space $V^{*}$.

Alternatively, one can define a representation in such a way that $h$ is a homomorphism and the dual action $G \times V^{*} \rightarrow V^{*}$ is defined by $(g \varphi)(v):=$ $\varphi\left(h\left(g^{-1}\right)(v)\right)$.

Remark 6.7. Let $X$ be a $G$-space and let $\mathcal{A}$ be a Banach (closed, unital) subalgebra of $C(X)$. Associated with $\mathcal{A}$ we have the canonical $\mathcal{A}$-compactification $\nu_{\mathcal{A}}: X \rightarrow X^{\mathcal{A}}$ of $X$, where $X^{\mathcal{A}}=|\mathcal{A}|$ is the Gelfand space of $\mathcal{A}$. Here $X^{\mathcal{A}}$ is canonically embedded into the weak* compact unit ball $B^{*}$ of the dual space $\mathcal{A}^{*}$. If $\mathcal{A}$ is $G$-invariant (that is, the function $(f g)(x):=f(g x)$ lies in $\mathcal{A}$ for every $f \in \mathcal{A}$ and $g \in G$ ) then $X^{\mathcal{A}}$ admits the natural adjoint action $G \times X^{\mathcal{A}} \rightarrow X^{\mathcal{A}}$ with the property that all translations $\breve{g}: X^{\mathcal{A}} \rightarrow X^{\mathcal{A}}$ are continuous and such that $\alpha_{\mathcal{A}}: X \rightarrow X^{\mathcal{A}} \subset B^{*}$ is $G$-equivariant. We obtain in this way a representation (where $h$ is not necessarily continuous)

$$
\left(h, \alpha_{\mathcal{A}}\right):(G, X) \rightrightarrows\left(\operatorname{Iso}(\mathcal{A}), B^{*}\right)
$$

on the Banach space $\mathcal{A}$, where $h(g)(f):=f g\left(\right.$ and $\left.\alpha_{\mathcal{A}}(x)(f):=f(x)\right)$. We call it the canonical (or regular) $\mathcal{A}$-representation of $(G, X)$. It is continuous iff $\mathcal{A} \subset R U C(X)$ (see for example [30, Fact 2.2] and [31, Fact 7.2]). The regular $R U C(X)$-representation leads to the maximal $G$-compactification $X \rightarrow \beta_{G} X$ of $X$. It is proper if and only if $X$ is $G$-compactifiable.

The following observation due to Teleman is well known (see also [38]).

FACT 6.8 (Teleman [43]). Every topological group can be embedded into Iso $(V)$ for some Banach space $V$.

Proof. It is well known that $R U C(G)$ determines the topology of $G$. Hence the regular $V:=R U C(G)$-representation $(h, \alpha):(G, G) \rightrightarrows\left(\operatorname{Iso}(V), B^{*}\right)$ is proper. That is, the map $\alpha$ is an embedding. In fact, it is easy to see that the co-homomorphism $h$ is an embedding of topological spaces. The representation $h^{\mathrm{op}}: G \rightarrow \operatorname{Iso}(V), g \mapsto h\left(g^{-1}\right)$, is then a topological group embedding.

Definition 6.9. Let $\mathcal{K}$ be a "well behaved" subclass of the class $\mathcal{B}$ an of all Banach spaces. Typical and important particular cases for such $\mathcal{K}$ are: $\mathcal{H}$ ilb, Ref or $\mathcal{A} s p$, the classes of Hilbert, reflexive or Asplund Banach spaces respectively. 
(1) A topological group $G$ is $\mathcal{K}$-representable if there exists a (co)representation $h: G \rightarrow \operatorname{Iso}(V)$ for some $V \in \mathcal{K}$ such that $h$ is topologically faithful (that is, an embedding). Notation: $G \in \mathcal{K}_{r}$.

(2) In the opposite direction, we say that $G$ is $\mathcal{K}$-trivial if every continuous $\mathcal{K}$-representation (or, equivalently, co-representation) $h: G \rightarrow$ Iso $(V)$ is trivial.

(3) We say that a topological group $G$ is $S U C$-trivial if $S U C(G)=$ \{constants\}. Analogously one can define WAP-trivial groups. $G$ is WAP-trivial iff $G$ is reflexively trivial ( $\mathcal{R} e f$-trivial in the sense of Definition 6.9(2)). Similarly, $\operatorname{Asp}(G)=\{$ constants $\}$ iff $G$ is $\mathcal{A} s p$-trivial. These equivalences follow for instance from Theorem 9.3 below.

REMARK 6.10.

(1) By Teleman's theorem (Fact 6.8) every topological group is "Banach representable". Hence, $\{$ Topological Groups $\}=\mathcal{B} a n_{r}$.

(2) $\{$ Topological Groups $\}={\mathcal{B} a n_{r}} \supset \mathcal{A}_{s p_{r}} \supset \mathcal{R e f}_{r} \supset \mathcal{H} i l b_{r}$.

(3) By Herer and Christensen [22] (see also Banaszczyk [4]) abelian (even monothetic) groups can be $\mathcal{H}$ ilb-trivial. Note also that $c_{0} \notin$ $\mathcal{H}$ Hilb $[29]$.

(4) The additive group $L_{4}[0,1]$ is reflexively but not Hilbert representable [29].

(5) $H_{+}[0,1] \notin \mathcal{R} f_{r}[28]$. It was shown in [28] that every weakly almost periodic function on the topological group $G:=H_{+}[0,1]$ is constant and that $G$ is Ref-trivial. By Pestov's observation (see [39, Corollary 1.4] and Lemma 10.2) the same is true for the group Iso $\left(\mathbb{U}_{1}\right)$.

(6) Theorem $10.3(3)$ shows that $H_{+}[0,1]$ is even $\mathcal{A} s p$-trivial. In fact we show that every "adjoint continuous" representation of that group is trivial (Theorem 10.3(2)). This result was obtained also by Uspenskij (unpublished). Furthermore, we prove a stronger result by showing that $H_{+}[0,1]$ (and also Iso $\left(\mathbb{U}_{1}\right)$ ) are SUC-trivial.

Problem 6.11 (see also [31] and [28]).

(1) Distinguish $\mathcal{A} s p_{r}$ and $\mathcal{R e f}_{r}$ by finding $G \in \mathcal{A} s p_{r}$ such that $G \notin \mathcal{R} e f_{r}$.

(2) Find an abelian $G \notin \operatorname{Ref}_{r}$.

Now we turn to the "well behaved actions". Recall the dynamical versions of Eberlein and Radon-Nikodým compact spaces.

Definition 6.12 ([13, 30]). Let $X$ be a $G$-space.

(1) $(G, X)$ is a Radon-Nikodym system (RN for short) if there exists a proper representation of $(G, X)$ on an Asplund Banach space $V$. If we can choose $V$ to be reflexive, then $(G, X)$ is called an Eberlein system. 
The classes of Radon-Nikodým and Eberlein compact systems will be denoted by $\mathrm{RN}$ and Eb respectively.

(2) $(G, X)$ is called an $R N$-approximable system $\left(\mathrm{RN}_{\text {app }}\right)$ if it can be represented as a subdirect product of RN systems.

Note that compact spaces which are not Eberlein are necessarily nonmetrizable, while even for $G:=\mathbb{Z}$, there are many natural metric compact $G$-systems which are not RN.

DEFINITION 6.13.

(1) A representation $(h, \alpha)$ of a $G$-space $X$ on $V$ is adjoint continuous if the dual action $G \times V^{*} \rightarrow V^{*}$ is also continuous (or, equivalently, if the group corepresentation $h: G \rightarrow \operatorname{Iso}(V)$ is adjoint continuous).

(2) Denote by $\mathcal{A} d j$ the class of compact $G$-systems which admit a proper adjoint continuous representation on some Banach space $V$. Theorem 6.5 implies that $\mathrm{RN} \subset \mathcal{A} d j$.

(3) Denote by $\operatorname{adj}(G)$ the collection of functions on $G$ which come from a compact $G$-space $X$ such that $(G, X)$ is in the class $\mathcal{A} d j$. In fact this means that $f$ can be represented as a generalized matrix coefficient (see Section 9) of some adjoint continuous representation of $G$.

Proposition 6.14. $\operatorname{Asp}(G) \subset \operatorname{adj}(G)$ for every topological group $G$.

Proof. By [30, Theorem 7.11] (or Proposition 7.5) $f \in \operatorname{Asp}(G)$ iff $f$ comes from a $G$-compactification $G \rightarrow X$ of $G$ with $X \in \mathrm{RN}$. Now observe (as in Definition 6.13.2) that $\mathrm{RN} \subset \mathcal{A} d j$ by Theorem 6.5.

7. Dynamical complexity of functions. In this section we introduce a hierarchy of dynamical complexity of functions on a topological group $G$ which reflects the complexity of the $G$-systems from which they come. Our main tool is the cyclic $G$-system $X_{f}$ corresponding to a function $f: X \rightarrow \mathbb{R}$. Recall that when $X:=G$, the space $X_{f}$ is the pointwise closure of the orbit $G f$ in $R U C(G)$. The topological nature of $X_{f}$ in the Banach space $R U C(G)$ relates to the dynamical complexity of $f$ and leads to a natural hierarchy of complexity (see Theorem 7.12 below). In particular, we will examine the role that SUC functions play in this hierarchy.

Periodic orbits and the profinite compactification. The most elementary dynamical system is a finite (periodic) orbit. It corresponds to a clopen subgroup $H<G$ of finite index. These subgroups form a directed set and the corresponding compact inverse limit $G$-system

$$
X^{P F}=\lim _{\longleftarrow} G / H
$$

is the profinite compactification of $G$. 
Almost periodic functions and the Bohr compactification. The weaker requirement that $X_{f}$ be norm compact in $R U C(G)$ leads to the well known definition of almost periodicity. A function $f \in C(X)$ on a $G$-space $X$ is almost periodic if the orbit $f G:=\{f g\}_{g \in G}$ forms a precompact subset of the Banach space $C(X)$. The collection $A P(X)$ of AP functions is a $G$ subalgebra in $R U C(X)$. The universal almost periodic compactification of $X$ is the Gelfand space $X^{A P}$ of the algebra $A P(X)$. When $X$ is compact this is the classical maximal equicontinuous factor of the system $X$. A compact $G$-space $X$ is equicontinuous iff $X$ is almost periodic (AP), that is, iff $C(X)=$ $A P(X)$. For a $G$-space $X$ the collection $A P(X)$ is the set of all functions which come from equicontinuous (AP) $G$-compactifications.

For every topological group $G$, treated as a $G$-space, the corresponding universal AP compactification is the well known Bohr compactification $b$ : $G \rightarrow b G$, where $b G$ is a compact topological group.

Theorem 7.1. Let $X$ be a $G$-space. For $f \in R U C(X)$ the following conditions are equivalent:

(1) $f \in A P(X)$.

(2) $\left(G, X_{f}\right)$ is equicontinuous.

(3) $X_{f}$ is norm compact in $R U C(G)$.

Proof. $(1) \Leftrightarrow(2): f \in A P(X)$ iff the cyclic algebra $\mathcal{A}_{f}$ (which, by Proposition 3.1, generates the compactification $\left.X \rightarrow X_{f}\right)$ is a subalgebra of $A P(X)$.

$(2) \Leftrightarrow(3)$ : It is easy to see that the $G$-space $X_{f}$ is equicontinuous iff the norm and pointwise topologies coincide on $X_{f} \subset R U C(G)$.

Weakly almost periodic functions. A function $f \in C(X)$ on a $G$-space $X$ is called weakly almost periodic (WAP for short; notation: $f \in W A P(X)$ ) if the orbit $f G:=\{f g\}_{g \in G}$ forms a weakly precompact subset of $C(X)$. A compact $G$-space $X$ is said to be weakly almost periodic [8] if $C(X)=$ $W A P(X)$. For a $G$-space $X$ the collection $W A P(X)$ is the set of all functions which come from WAP $G$-compactifications. The universal WAP $G$ compactification $X \rightarrow X^{W A P}$ is well defined. The algebra $W A P(G)$ is a point-universal $G$-algebra containing $A P(G)$. The compactification $G \rightarrow$ $G^{W A P}$ (for $X:=G$ ) is the universal semitopological semigroup compactification of $G$.

A compact $G$-space $X$ is WAP iff it admits sufficiently many representations on reflexive Banach spaces [30]. Furthermore, if $X$ is a metric compact $G$-space then $X$ is WAP iff $X$ admits a proper representation on a reflexive Banach space. That is, iff $X$ is an Eberlein $G$-space.

Theorem 7.2. Let $X$ be a $G$-space. For $f \in R U C(X)$ the following conditions are equivalent:

(1) $f \in W A P(X)$. 
(2) $\left(G, X_{f}\right)$ is WAP.

(3) $X_{f}$ is weak compact in $R U C(G)$.

(4) $\left(G, X_{f}\right)$ is Eberlein (i.e., reflexively representable).

Proof. (1) $\Leftrightarrow(2): f \in W A P(X)$ iff the algebra $\mathcal{A}_{f}$ is a subalgebra of $W A P(X)$.

$(2) \Rightarrow(3):$ Let $F_{e}: X_{f} \rightarrow \mathbb{R}, F_{e}(\omega)=\omega(e)$ be as in the definition of $X_{f}$. Consider the weak closure $Y:=\operatorname{cl}_{w}\left(F_{e} G\right)$ of the orbit $F_{e} G$. Then $Y$ is weakly compact in $C\left(X_{f}\right)$ because $F_{e} \in C\left(X_{f}\right)=W A P\left(X_{f}\right)$ is weakly almost periodic. If $\omega_{1}$ and $\omega_{2}$ are distinct elements of $X_{f}$ then $\left(F_{e} g\right)\left(\omega_{1}\right)=$ $\omega_{1}(g) \neq \omega_{2}(g)=\left(F_{e} g\right)\left(\omega_{2}\right)$ for some $g \in G$. This means that the separately continuous evaluation map $Y \times X_{f} \rightarrow \mathbb{R}$ separates points of $X_{f}$. Now $X_{f}$ can be treated as a pointwise compact bounded subset in $C(Y)$. Hence by Grothendieck's well known theorem [21] we find that $X_{f}$ is weakly compact in $C(Y)$. Since $G \rightarrow Y, g \mapsto g F_{e}$, is a $G$-compactification of $G$, we have a natural embedding of Banach algebras $j: C(Y) \hookrightarrow R U C(G)$. It follows that $X_{f}=j\left(X_{f}\right)$ is also weakly compact as a subset of $R U C(G)$.

$(3) \Rightarrow(4)$ : The isometric action $G \times R U C(G) \rightarrow R U C(G),(g, f) \mapsto g f$, induces a representation $h: G \rightarrow \operatorname{Iso}(R U C(G))$. If the $G$-subset $X_{f}$ is weakly compact in $R U C(G)$ then one can apply Theorem 4.11 (namely, the equivalence between (i) and (ii)) of [30] which guarantees that the $G$-space $X_{f}$ is Eberlein.

$(4) \Rightarrow(1): f \in W A P(X)$ because it comes from $\left(G, X_{f}\right)$ (Proposition 3.1) which is WAP (being reflexively representable).

Asplund functions, "sensitivity to initial conditions" and Banach representations. The following definition of "sensitivity to initial conditions" is essential in several definitions of chaos in dynamical systems, mostly for $G:=\mathbb{Z}$ or $\mathbb{R}$ actions on metric spaces (see for instance papers of Guckenheimer, Auslander and Yorke, Devaney, Glasner and Weiss).

Definition $7.3([13])$. Let $(X, \mu)$ be a uniform $G$-space.

(1) We say that $X$ is sensitive to initial conditions (or just sensitive) if there exists an $\varepsilon \in \mu$ such that for every nonempty open subset $O$ of $X$ the set $g O$ is not $\varepsilon$-small for some $g \in G$. Otherwise, $X$ is nonsensitive (for short: NS).

(2) $X$ is hereditarily non sensitive (HNS) if every closed $G$-subspace of $X$ is NS.

Denote by HNS the class of all compact HNS systems. The following result says that a compact $G$-system $X$ is $\operatorname{HNS}$ iff $(G, X)$ admits sufficiently many representations on Asplund spaces. 
THEOREM 7.4 ([13]).

(1) $\mathrm{HNS}=\mathrm{RN}_{\mathrm{app}}$.

(2) If $X$ is a compact metric G-space then $X$ is HNS iff $X$ is RN (that is, Asplund representable).

A function $f: X \rightarrow \mathbb{R}$ on a $G$-space $X$ is Asplund (notation: $f \in \operatorname{Asp}(X)$ ) $[30]$ if it satisfies one of the following equivalent conditions.

Proposition 7.5. Let $f: X \rightarrow \mathbb{R}$ be a function on a $G$-space $X$. The following conditions are equivalent:

(1) $f$ comes from a $G$-compactification $\nu: X \rightarrow Y$ where $(G, Y)$ is HNS.

(2) $f$ comes from a $G$-compactification $\nu: X \rightarrow Y$ where $(G, Y)$ is $R N$.

(3) $f$ comes from a $G$-compactification $\nu: X \rightarrow Y$ and a function $F$ : $Y \rightarrow \mathbb{R}$ where the pseudometric space $\left(Y, \varrho_{H, F}\right)$ with

$$
\varrho_{H, F}\left(x, x^{\prime}\right)=\sup _{h \in H}\left|F(h x)-F\left(h x^{\prime}\right)\right| .
$$

is separable for every countable (equivalently, second countable) subgroup $H \subset G$.

The collection $\operatorname{Asp}(X)$ is always a $G$-subalgebra of $R U C(X)$. It defines the maximal HNS-compactification $X \rightarrow X^{\operatorname{Asp}}=|\operatorname{Asp}(X)|$ of $X$. For every topological group $G$ the algebra $\operatorname{Asp}(G)$ (as usual, $X:=G$ is a left $G$-space) is point-universal.

TheOrem 7.6. Let $X$ be a $G$-space. For every $f \in R U C(X)$ the following conditions are equivalent:

(1) $f \in \operatorname{Asp}(X)$.

(2) $\left(G, X_{f}\right)$ is $R N$.

(3) $X_{f}$ is norm fragmented in $R U C(G)$.

Proof. If $X$ is compact then the proof follows directly from [13, Theorem 9.12]. Now observe that one can reduce the case of an arbitrary $G$-space $X$ to the case of a compact $G$-space $X_{f}$ by considering the cyclic $G$-system $\left(X_{f}\right)_{F_{e}}$ (defined for $X:=X_{f}$ and $f:=F_{e}$ ) which can be naturally identified with $X_{f}$.

Explicitly the fragmentability of $X_{f}$ means that for every $\varepsilon>0$ every nonempty (closed) subset $A$ of $X_{f} \subset \mathbb{R}^{G}$ contains a relatively open (in the pointwise topology) nonempty subset $O \cap A$ which is $\varepsilon$-small in the Banach space $R U C(G)$.

As already mentioned, every weakly compact set is norm fragmented so that $W A P(X) \subset \operatorname{Asp}(X)$ for every $G$-space $X$. In particular, $W A P(G) \subset$ $\operatorname{Asp}(G)$. 
Locally equicontinuous functions. During the last decade various conditions weakening the classical notion of equicontinuity were introduced and studied (see e.g. [16], [1], [2], [3]). The following definition first appears in a paper of Glasner and Weiss [16].

Definition $7.7([16])$. Let $(X, \mu)$ be a uniform $G$-space. A point $x_{0} \in X$ is a point of local equicontinuity (notation: $x_{0} \in L E_{X}$ ) if $x_{0}$ is a point of equicontinuity in the uniform $G$-subspace $\operatorname{cl}\left(G x_{0}\right)$. We have $x_{0} \in L E_{X}$ iff $x_{0} \in L E_{Y}$ iff $x_{0} \in \mathrm{Eq}_{Y}$ where $Y$ is the orbit $G x_{0}$ of $x_{0}$ (see Lemma 7.8(1)). If $L E_{X}=X$, then $X$ is locally equicontinuous (LE).

LEMMA 7.8.

(1) Let $Y$ be a dense $G$-subspace of $(X, \mu)$ and $y_{0} \in Y$. Then $y_{0} \in \mathrm{Eq}_{X}$ if and only if $y_{0} \in \mathrm{Eq}_{Y}$.

(2) $S U C_{X} \supset L E_{X} \supset \mathrm{Eq}_{X}$.

(3) $\operatorname{SUC}(X) \supset L E(X)$.

Proof. (1): Let $\varepsilon \in \mu$. There exists $\delta \in \mu$ such that $\delta$ is a closed subset of $X \times X$ and $\delta \subset \varepsilon$. If $y_{0} \in \mathrm{Eq}_{Y}$ there exists an open set $U$ in $X$ such that $y_{0} \in U$ and $\left(g y, g y_{0}\right) \in \delta$ for all $y \in U \cap Y$ and $g \in G$. Since $Y$ is dense in $X$ and $U$ is open we have $U \subset \operatorname{cl}(U \cap Y)$. Since every $g$-translation $X \rightarrow X, x \mapsto g x$, is continuous and $\delta$ is closed we get $\left(g x, g y_{0}\right) \in \delta \subset \varepsilon$ for every $g \in G$ and $x \in U$.

(2): Let $x_{0} \in L E_{X}$. For every $\varepsilon \in \mu$ there exists a neighborhood $O\left(x_{0}\right)$ such that $\left(g x, g x_{0}\right)$ is $\varepsilon$-small for every $x \in O\left(x_{0}\right)$ and $g \in G$. Choose a neighborhood $U(e)$ such that $U x_{0} \subset O$. Then $\left(g u x_{0}, g x_{0}\right)$ is also $\varepsilon$-small. This proves the nontrivial part $S U C_{X} \supset L E_{X}$.

(3): Directly follows from (2).

The collection $L E(X)$ forms a $G$-subalgebra of $R U C(X)$. Always, $\operatorname{Asp}(X)$ $\subset L E(X)$. The algebra $L E(X)$ defines the maximal LE-compactification $X \rightarrow X^{L E}$ of $X$. For every topological group $G$ the algebra $L E(G)$ is pointuniversal [13].

Theorem 7.9 ([13]). Let $X$ be a $G$-space. For $f \in R U C(X)$ the following conditions are equivalent:

(1) $f \in L E(X)$.

(2) $\left(G, X_{f}\right)$ is $L E$.

(3) $X_{f}$ is orbitwise light in $R U C(G)$ (that is, for every function $\psi \in X_{f}$ the pointwise and norm topologies coincide on the orbit $G \psi$ ).

Proof. $(1) \Leftrightarrow(2)$ : Directly follows from [13, Theorem 5.15.1]. On the other hand, by [13, Lemma 5.18] we have $(2) \Leftrightarrow(3)$. 
The dynamical hierarchy

THEOREM 7.10.

(1) Let $X$ be a (not necessarily compact) G-space. We have the following chain of inclusions of $G$-subalgebras:

$R U C(X) \supset S U C(X) \supset L E(X) \supset \operatorname{Asp}(X) \supset W A P(X) \supset A P(X)$

and the corresponding chain of $G$-factor maps

$$
\beta_{G} X \rightarrow X^{S U C} \rightarrow X^{L E} \rightarrow X^{\text {Asp }} \rightarrow X^{W A P} \rightarrow X^{A P} .
$$

(2) For every topological group $G$ we have the following chain of inclusions of $G$-subalgebras:

$$
\begin{aligned}
R U C(G) \supset U C(G) \supset S U C(G) & \supset L E(G) \\
& \supset \operatorname{Asp}(G) \supset W A P(G) \supset A P(G)
\end{aligned}
$$

and the corresponding chain of $G$-factor maps

$$
G^{R U C} \rightarrow G^{U C} \rightarrow G^{S U C} \rightarrow G^{L E} \rightarrow G^{\mathrm{Asp}} \rightarrow G^{W A P} \rightarrow G^{A P} .
$$

Proof. For the assertions concerning $S U C(X)$ and $S U C(G)$ see Lemmas 4.3 and 7.8. For the other assertions see [13].

REMARK 7.11. The compactifications $G^{A P}$ and $G^{W A P}$ of $G$ are respectively a topological group and a semitopological semigroup. The compactifications $G^{R U C}$ and $G^{\text {Asp }}$ are right topological semigroup compactifications of $G$ (see [13]). The same is true for the compactification $j: G \rightarrow G^{S U C}$ (Lemma 4.6(5)). Below (Theorem 10.3(5)) we show that the Roelcke compactification $i: G \hookrightarrow G^{U C}$ (which is always proper by Lemma 2.1) is not in general a right topological semigroup compactification. That is, $U C(G)$ is not in general point-universal.

We sum up our results in the following dynamical hierarchy theorem where we list dynamical properties of $f \in R U C(X)$ and the corresponding topological properties of $X_{f} \subset R U C(G)$ (cf. [13, Remark 9.13]).

Theorem 7.12. For every $G$-space $X$ and a function $f \in R U C(X)$ we have

$X_{f}$ is norm compact $\Leftrightarrow f$ is $A P$,

$X_{f}$ is weakly compact $\Leftrightarrow f$ is WAP,

$X_{f}$ is norm fragmented $\Leftrightarrow f$ is Asplund,

$X_{f}$ is orbitwise light $\Leftrightarrow f$ is $L E$,

$X_{f} \subset U C(G) \Leftrightarrow f$ is $S U C$.

EXAmPLE 7.13. Let $G$ be the unitary group $U(H)=\operatorname{Iso}(H)$ where $H$ is an infinite-dimensional Hilbert space. Then $U C(G)=S U C(G)=L E(G)=$ $\operatorname{Asp}(G)=W A P(G)$. Indeed the completion of $(G, \mathcal{L} \wedge \mathcal{R})$ can be identified 
with the compact semitopological semigroup $\Theta(H)$ of all nonexpansive linear operators (Uspenskij [46]). It follows that $G^{U C}$ can be identified with $\Theta(H)$. The latter is a reflexively representable $G$-space (see for example [30, Fact 5.2]). Therefore $U C(G) \subset W A P(G)$. The reverse inclusion is well known (see for instance Theorem 7.10). Hence, $U C(G)=W A P(G)$.

Let $\mathrm{C}_{u}=\left\{f \in U C(G): X_{f} \subset U C(G)\right\}$. The collection of functions $\mathrm{C}_{u}$ was studied by Veech in [49]. He notes there that $W A P(G) \subset \mathcal{C}_{u}$ and proves the following theorem.

TheOREM 7.14 (Veech [49, Proposition 5.4]). Let $G$ be a semisimple analytic Lie group with finite center and without compact factors. If $f \in \mathrm{C}_{u}$ then every limit point of $G f$ in $X_{f}$, i.e. any function of the form $h(g)=$ $\lim _{g_{n} \rightarrow \infty} f\left(g g_{n}\right)$, is a constant function.

(A sequence $g_{n} \in G$ "tends to $\infty$ " if each of its "projections" onto the simple components of $G$ tends to $\infty$ in the usual sense.) He then deduces the fact that for $G$ which is a direct product of simple groups the algebra $W A P(G)$ coincides with the algebra $\mathcal{W}^{*}$ of continuous functions on $G$ which "extend continuously" to the product of the one-point compactifications of the simple components of $G$ ([49, Theorem 1.2]). By our Proposition 4.12, $\mathrm{C}_{u}=S U C(G)$. Taking this equality into account, Veech's theorem now implies the following result.

COROLlary 7.15. For every simple noncompact connected Lie group $G$ with finite center $\left(\right.$ e.g., $\left.S L_{n}(\mathbb{R})\right)$ we have $S U C(G)=W A P(G)=\mathcal{W}^{*}$. In particular, the corresponding universal SUC (and hence WAP) compactification is equivalent to the one-point compactification of $G$.

8. The group $H_{+}[0,1]$. Consider the Polish topological group $G:=$ $H_{+}[0,1]$ of all orientation preserving homeomorphisms of the closed unit interval, endowed with the compact-open topology. Here is a list of some selected known results about this group:

(1) $G$ is topologically simple.

(2) $G$ is not Weil-complete; that is, the right uniform structure $\mathcal{R}$ of $G$ is not complete. The completion of the uniform space $(G, \mathcal{R})$ can be identified with the semigroup of all continuous, nondecreasing and surjective maps $[0,1] \rightarrow[0,1]$ endowed with the uniform structure of uniform convergence (Roelcke-Dierolf [41, p. 191]).

(3) $G$ is Roelcke precompact (that is, the Roelcke uniformity $\mathcal{L} \wedge \mathcal{R}$ on $G$ is precompact) [41].

(4) The completion of $(G, \mathcal{L} \wedge \mathcal{R})$ can be identified with the curves that connect the points $(0,0)$ and $(1,1)$ and "never go down" (Uspenskij [48], see Lemma 8.4 below). 
(5) Every weakly almost periodic function on $G$ is constant and every continuous representation $G \rightarrow \operatorname{Iso}(V)$, where $V$ is a reflexive Banach space, is trivial (Megrelishvili [28]).

(6) $G$ is extremely amenable; that is, every compact Hausdorff $G$-space has the fixed point property (Pestov [37]).

We are going to show that $H_{+}[0,1]$ is SUC-trivial and hence also $\mathcal{A} s p$ trivial. Since every reflexive Banach space is Asplund these results strengthen the main results of [28] (results mentioned in item (5) above).

Definition 8.1. Let $(X, \mu)$ be a compact $G$-space. We say that two points $a, b \in X$ are $S U C$-proximal if there exist nets $s_{i}$ and $g_{i}$ in $G$ and a point $x_{0} \in X$ such that $s_{i}$ converges to the neutral element $e$ of $G$, the net $g_{i} x_{0}$ converges to $a$ and the net $g_{i} s_{i} x_{0}$ converges to $b$.

Lemma 8.2. If the points $a$ and $b$ are $S U C$-proximal in a $G$-space $X$ then $a \stackrel{S U C}{\sim} b$.

Proof. A straightforward consequence of our definitions using Lemma 4.5.4.

THEOREM 8.3. Let $G=H_{+}[0,1]$ be the topological group of orientationpreserving homeomorphisms of $[0,1]$ endowed with the compact-open topology. Then $G$ is SUC-trivial.

Proof. Denote by $j: G \rightarrow G^{S U C}$ and $i: G \rightarrow G^{U C}$ the $G$-compactifications ( $i$ necessarily is proper by Lemma 2.1) induced by the Banach $G$ algebras $S U C(G) \subset U C(G)$. There exists a canonical onto $G$-map $\pi$ : $G^{U C} \rightarrow G^{S U C}$ such that the following diagram of $G$-maps is commutative:

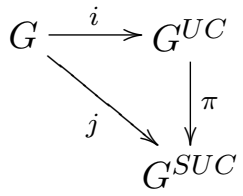

We have to show that $G^{S U C}$ is trivial for $G=H_{+}[0,1]$. One of the main tools for the proof is the following identification.

LEMma 8.4 (Uspenskij [48]). The dynamical system $G^{U C}$ is isomorphic to the $G$-space $(G, \Omega)$. Here $\Omega$ denotes the compact space of all curves in $[0,1] \times[0,1]$ which connect the points $(0,0)$ and $(1,1)$ and "never go down", equipped with the Hausdorff metric. These are the relations $\omega \subset[0,1] \times$ $[0,1]$ where for each $t \in[0,1], \omega(t)$ is either a point or a vertical closed segment. The natural action of $G=H_{+}[0,1]$ on $\Omega$ is $(g \omega)(t)=g(\omega(t))$ (by composition of relations on $[0,1])$. 
We first note that every "zig-zag curve" (i.e. a curve $z$ which consists of a finite number of horizontal and vertical pieces) is an element of $\Omega$. In particular, the curves $\gamma_{c}$ with exactly one vertical segment, defined as $\gamma_{c}(t)=0$ for every $t \in[0, c), \gamma_{c}(c)=\{c\} \times[0,1]$ and $\gamma(t)=1$ for every $t \in(c, 1]$, are elements of $\Omega=G^{U C}$. Note that the curve $\gamma_{1}$ is a fixed point for the left $G$-action. We let $\theta=\pi\left(\gamma_{1}\right)$ be its image in $G^{S U C}$. Of course $\theta$ is a fixed point in $G^{S U C}$. We will show that $\theta=j(e)$ and since the $G$-orbit of $j(e)$ is dense in $G^{S U C}$ this will show that $G^{S U C}$ is a singleton.

The idea is to show that zig-zag curves are SUC-proximal in $G^{U C}$. Then Lemma 8.2 will ensure that their images in $G^{S U C}$ coincide. Choosing a sequence $z_{n}$ of zig-zag curves which converges in the Hausdorff metric to $i(e)$ in $G^{U C}$ we will have $\pi\left(z_{n}\right)=\pi\left(\gamma_{1}\right)=\theta$ for each $n$. This will imply that indeed $j(e)=\pi(i(e))=\pi\left(\lim _{n \rightarrow \infty} z_{n}\right)=\lim _{n \rightarrow \infty} \pi\left(z_{n}\right)=\theta$.

First we show that $\pi\left(\gamma_{1}\right)=\pi\left(\gamma_{c}\right)$ for any $0<c<1$. As indicated above, since $G^{S U C}$ is the Gelfand space of the algebra $S U C(G)$, by Lemma 8.2 it suffices to show that the pair $\gamma_{1}, \gamma_{c}$ is SUC-proximal in $G^{U C}$. Since $X^{S U C}=X$ for $X:=G^{S U C}$, we conclude that $\pi\left(\gamma_{1}\right)=\pi\left(\gamma_{c}\right)$.

Let $p \in G^{U C}$ be the curve defined by $p(t)=t$ in the interval $[0, c]$ and by $p(t)=c$ for every $t \in[c, 1)$. Pick a sequence $s_{n}$ of elements in $G$ such that $s_{n}$ converges to $e$ and $s_{n} c<c$. It is easy to choose a sequence $g_{n}$ in $G$ such that $g_{n} s_{n} c$ converges to 0 and $g_{n} c$ converges to 1 . Then the sequences $s_{n}$ and $g_{n}$ are as desired, that is, $g_{n} p \rightarrow \gamma_{c}, g_{n} s_{n} p \rightarrow \gamma_{1}$ (see the picture below).
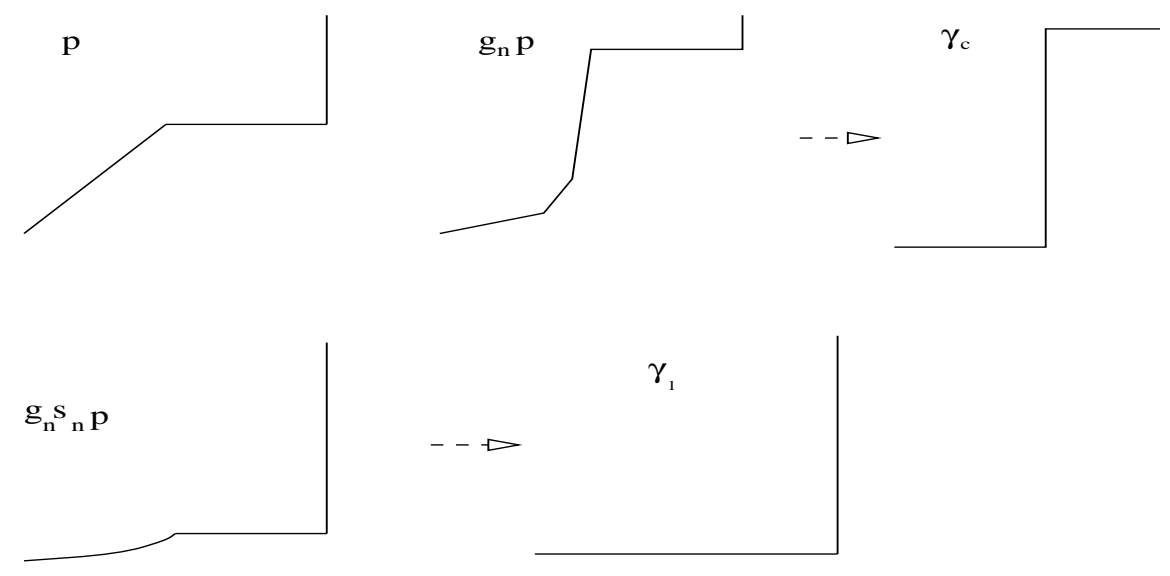

Set $\theta=\pi\left(\gamma_{1}\right)=\pi\left(\gamma_{c}\right)$. Using similar arguments (see the picture below, where $a \stackrel{\pi}{\sim} b$ means $\pi(a)=\pi(b))$ construct a sequence $z_{n} \in G^{U C}$ of zig-zag curves which converges to $i(e)$ and such that $\pi\left(z_{n}\right)=\theta$ for every $n$. 


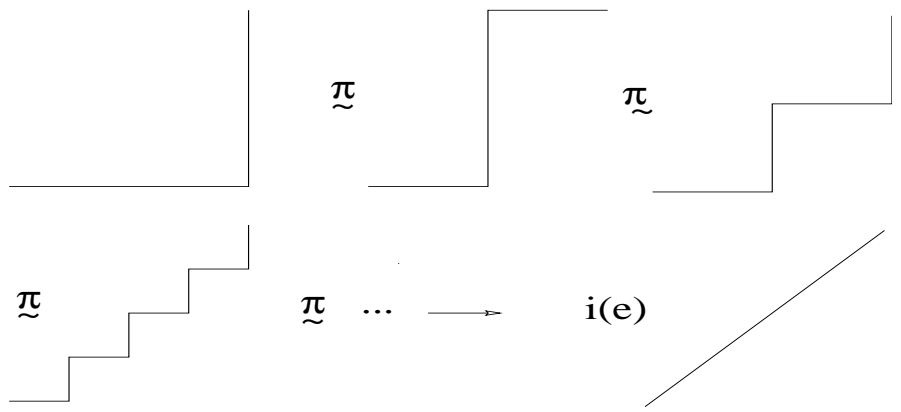

In view of the discussion above this construction completes the proof of the theorem.

\section{Matrix coefficient characterization of SUC and LE}

Definition 9.1. Let $h: G \rightarrow \operatorname{Iso}(V)$ be a co-representation of $G$ on a normed space $V$ and let

$$
V \times G \rightarrow V, \quad(v, g) \mapsto v g:=h(g)(v),
$$

be the corresponding right action. For a pair of vectors $v \in V$ and $\psi \in V^{*}$ the associated matrix coefficient is defined by

$$
m_{v, \psi}: G \rightarrow \mathbb{R}, \quad g \mapsto \psi(v g)=\langle v g, \psi\rangle=\langle v, g \psi\rangle .
$$

If $h: G \rightarrow \operatorname{Iso}(V)$ is a representation then the matrix coefficient $m_{v, \psi}$ is defined similarly by

$$
m_{v, \psi}: G \rightarrow \mathbb{R}, \quad g \mapsto \psi(g v)=\langle g v, \psi\rangle=\langle v, \psi g\rangle .
$$

For example, if $V=H$ is a Hilbert space then $f=m_{u, \psi}$ is the FourierStieltjes transform. In particular, for $u=\psi$ we get the positive definite functions.

We say that a vector $v \in V$ is $G$-continuous if the corresponding orbit map $\widetilde{v}: G \rightarrow V, \widetilde{v}(g)=v g$, defined through $h: G \rightarrow \operatorname{Iso}(V)$, is norm continuous. The continuous $G$-vectors $\psi \in V^{*}$ are defined similarly with respect to the dual action.

Lemma 9.2 ([30]). Let $h: G \rightarrow \operatorname{Iso}(V)$ be a co-representation of $G$ on $V$. If $\psi$ (resp. $v \in V)$ is norm $G$-continuous, then $m_{v, \psi}$ is left (resp. right) uniformly continuous on $G$. Hence, if $v$ and $\psi$ are both $G$-continuous then $m_{v, \psi} \in U C(G)$.

In the next theorem we list characterizations of several subalgebras of $R U C(G)$ in terms of matrix coefficients. These characterizations also provide an alternative way to establish the inclusions in Theorem 7.10(2) for $X:=G$.

Theorem 9.3. Let $G$ be a topological group and $f \in C(G)$.

(1) $f \in R U C(G)$ iff $f=m_{v, \psi}$ for some continuous co-representation $h: G \rightarrow \operatorname{Iso}(V)$, where $V \in \mathcal{B}$ an. 
(2) $f \in U C(G)$ iff $f=m_{v, \psi}$ for some co-representation where $v$ and $\psi$ are both $G$-continuous iff $f=m_{v, \psi}$ for some continuous co-representation where $\psi$ is $G$-continuous.

(3) $f \in S U C(G)$ iff $f=m_{v, \psi}$ for some continuous co-representation $h: G \rightarrow \operatorname{Iso}(V)$, where $\varphi$ is norm $G$-continuous in $V^{*}$ for every $\varphi$ from the weak $k^{*}$ closure $\mathrm{cl}_{w^{*}}(G \psi)$.

(4) $f \in \operatorname{adj}(G)$ iff $f=m_{v, \psi}$ for some adjoint continuous co-representation.

(5) $f \in L E(G)$ iff $f=m_{v, \psi}$ for some continuous co-representation $h$ : $G \rightarrow \operatorname{Iso}(V)$, where weak ${ }^{*}$ and norm topologies coincide on each orbit $G \varphi$ where $\varphi$ belongs to the weak ${ }^{*}$ closure $Y:=\operatorname{cl}_{w^{*}}(G \psi)$.

(6) $f \in \operatorname{Asp}(G)$ iff $f$ is a matrix coefficient of some continuous Asplund co-representation of $G$.

(7) $f \in W A P(G)$ iff $f$ is a matrix coefficient of some continuous reflexive co-representation (or representation) of $G$.

Proof. Claim (1) follows by taking in the regular $R U C(G)$-corepresentation $V:=R U C(G), v:=f$ and $\psi:=\alpha(e)$. Claim (4) is a reformulation of Definition 6.13(3).

The remaining assertions are essentially nontrivial. Their proofs are based on an equivariant generalization of the Davis-Figiel-JohnsonPełczyński interpolation technique [7]. For detailed proofs of (6) and (7) (for co-representations) see [30, Theorem 7.17] and [30, Theorem 5.1]. As to the "representations case" in (7) observe that a matrix coefficient of a (continuous) co-representation on a reflexive space $V$ can be treated as matrix coefficient of a (continuous) representation on the dual space $V^{*}$. The continuity of the dual action follows by Theorem 6.5.

For (2) see [32]. Below we provide the proof of the new assertions (3) and (5). See Theorems 9.8 and 9.10 respectively.

REMARK 9.4. Let $\mathcal{A} \subset R U C(G)$ be a point-universal $G$-subalgebra. Then $\mathcal{A}$ is left m-introverted in the sense of [33], [5, Definition 1.4.11]. Indeed, we have only to check that every matrix coefficient $m_{v, \psi}$ of the regular $\mathcal{A}$-representation of the action $(G, G)$ on the Banach space $\mathcal{A}$ belongs again to $\mathcal{A}$ whenever $v \in \mathcal{A}$ and $\psi \in|\mathcal{A}| \subset \mathcal{A}^{*}$. Let $X:=|\mathcal{A}|$. Then the $G$-system $(X$, eva $(e))$ is point-universal and $\mathcal{A}=\mathcal{A}(X$, eva $(e))$. The matrix coefficient $m_{v, \psi}$ comes from the subsystem $(\operatorname{cl}(G \psi), \psi)$. In other words $m_{v, \psi} \in \mathcal{A}(\operatorname{cl}(G \psi), \psi)$. By Lemma 3.3 we have $\mathcal{A}(\operatorname{cl}(G \psi), \psi) \subset \mathcal{A}(X$, eva $(e))$. Thus $m_{v, \psi} \in \mathcal{A}(X$, eva $(e))=\mathcal{A}$.

DeFinition 9.5.

(1) Let $G$ be a topological group and $G \times X \rightarrow X$ and $Y \times G \rightarrow Y$ be respectively left and right actions. A map $\langle\rangle:, Y \times X \rightarrow \mathbb{R}$ is 
G-compatible if

$$
\langle y g, x\rangle=\langle y, g x\rangle \quad \forall(y, g, x) \in Y \times G \times X .
$$

(2) We say that a subset $M \subset Y$ is $S U C$-small at $x_{0} \in X$ if for every $\varepsilon>0$ there exists a neighborhood $U$ of $e$ such that

$$
\left|\left\langle v, u x_{0}\right\rangle-\left\langle v, x_{0}\right\rangle\right| \leq \varepsilon \quad \forall(v, u) \in M \times U .
$$

If $M$ is SUC-small at every $x \in X$ then we say that $M$ is $S U C$-small for $X$.

(3) Let $h: G \rightarrow \operatorname{Iso}(V)$ be a continuous co-representation on a normed space $V$ and $h^{*}: G \rightarrow \operatorname{Iso}\left(V^{*}\right)$ be the dual representation. Then we say that $M \subset V$ is $S U C$-small at $x_{0} \in X \subset V^{*}$ if this happens in the sense of (2) regarding the canonical bilinear $G$-compatible map $\langle\rangle:, V \times V^{*} \rightarrow \mathbb{R}$.

For example, a vector $\psi \in V^{*}$ in the dual space $V^{*}$ is $G$-continuous iff the unit ball $B_{V}$ of $V$ is SUC-small at $\psi$ (see Lemma 9.7(3)).

We give some useful properties of SUC-smallness.

LEMMA 9.6.

(1) Let $Y_{1} \times X_{1} \rightarrow \mathbb{R}$ and $Y_{2} \times X_{2} \rightarrow \mathbb{R}$ be two G-compatible maps. Suppose that $\gamma_{1}: X_{1} \rightarrow X_{2}$ and $\gamma_{2}: Y_{2} \rightarrow Y_{1}$ are $G$-maps such that

$$
\left\langle y, \gamma_{1}(x)\right\rangle=\left\langle\gamma_{2}(y), x\right\rangle \quad \forall(y, x) \in Y_{2} \times X_{1} .
$$

Then for every nonempty subset $M \subset Y_{2}$ the subset $\gamma_{1}(M) \subset Y_{1}$ is $S U C$-small at $x \in X_{1}$ if and only if $M$ is $S U C$-small at $\gamma_{1}(x) \in X_{2}$.

(2) Let $X$ be a (not necessarily compact) $G$-space. If $f \in S U C(X)$ then:

(a) $f G$ is $S U C$-small for $X$ with respect to the $G$-compatible evaluation map

$$
f G \times X \rightarrow \mathbb{R}, \quad(f g, x) \mapsto f(g x) .
$$

(b) The subset $F_{e} G$ of $C\left(X_{f}\right)$ is $S U C$-small for $X_{f}$ considered as a subset of $V^{*}$ where $V:=C\left(X_{f}\right)$ (with respect to the canonical map $V \times V^{*} \rightarrow \mathbb{R}$ and the natural co-representation $G \rightarrow \operatorname{Iso}(V)$ ).

Proof. (1): Observe that for every triple $\left(m, u, x_{0}\right) \in M \times G \times X$ we have

$$
\begin{aligned}
\left\langle\gamma_{1}(m), u x_{0}\right\rangle-\left\langle\gamma_{1}(m), x_{0}\right\rangle & =\left\langle m, \gamma_{2}\left(u x_{0}\right)\right\rangle-\left\langle m, \gamma_{2}\left(x_{0}\right)\right\rangle \\
& =\left\langle m, u \gamma_{2}\left(x_{0}\right)\right\rangle-\left\langle m, \gamma_{2}\left(x_{0}\right)\right\rangle .
\end{aligned}
$$

(2)(a): Directly follows by Lemma 4.5(4).

(3)(b): Let $f \in S U C(X)$. Then by Proposition 3.1(3) it comes from a compact $G$-system $X_{f}$ and the $G$-compactification $f_{\sharp}: X \rightarrow X_{f}$. We know that there exists $F_{e} \in C\left(X_{f}\right)$ such that $f=F_{e} \circ f_{\sharp}$. Theorem 4.12 implies that $X_{f}$ is SUC and $F_{e} \in S U C\left(X_{f}\right)$. By claim (a) it follows that $F_{e} G$ is SUC-small for $X_{f}$. The $G$-compatible map $F_{e} G \times X_{f} \rightarrow \mathbb{R}$ can be treated as 
a restriction of the canonical form $V \times V^{*} \rightarrow \mathbb{R}$, where $V:=C\left(X_{f}\right)$ (with $X_{f}$ considered as a subset of $\left.C\left(X_{f}\right)^{*}\right)$.

LEMma 9.7. Let $h: G \rightarrow \operatorname{Iso}(V)$ be a continuous co-representation.

(1) For every subset $X$ of $V^{*}$ the family of SUC-small sets for $X$ in $V$ is closed under taking subsets, norm closures, finite linear combinations, finite unions and convex hulls.

(2) If $M_{n} \subset V$ is SUC-small at $x_{0} \in V^{*}$ for every $n \in \mathbb{N}$ then so is the set $\bigcap_{n \in \mathbb{N}}\left(M_{n}+\delta_{n} B_{V}\right)$ for every positive decreasing sequence $\delta_{n} \rightarrow 0$.

(3) For every $\psi \in V^{*}$ the following are equivalent:

(i) The orbit map $\widetilde{\psi}: G \rightarrow V^{*}$ is norm continuous.

(ii) $\mathcal{B}$ is SUC-small at $\psi$, where $\mathcal{B}:=\left\{\check{v}: V^{*} \rightarrow \mathbb{R}, x \mapsto \check{v}(x):=\right.$ $\langle v, x\rangle\}_{v \in B_{V}}$.

Proof. Assertion (1) is straightforward.

(2): We have to show that the set $\bigcap_{n \in \mathbb{N}}\left(M_{n}+\delta_{n} B_{V}\right)$ is SUC-small at $x_{0}$. Let $\varepsilon>0$ be fixed. Since $G x_{0}$ is a bounded subset of $V^{*}$ one can choose $n_{0} \in \mathbb{N}$ such that $\left|v\left(g x_{0}\right)\right|<\varepsilon / 4$ for every $g \in G$ and every $v \in \delta_{n_{0}} B_{V}$. Since $M_{n_{0}}$ is SUC-small at $x_{0}$ we can choose a neighborhood $U(e)$ such that $\left|m\left(u x_{0}\right)-m\left(x_{0}\right)\right|<\varepsilon / 2$ for all $u \in U$ and $m \in M_{n_{0}}$. Now every element $w \in \bigcap_{n \in \mathbb{N}}\left(M_{n}+\delta_{n} B_{V}\right)$ has the form $w=m+v$ for some $m \in M_{n_{0}}$ and $v \in \delta_{n_{0}} B_{V}$. Then for every $u \in U$ we have

$\left|w\left(u x_{0}\right)-w\left(x_{0}\right)\right| \leq\left|m\left(u x_{0}\right)-m\left(x_{0}\right)\right|+\left|v\left(u x_{0}\right)\right|+\left|v\left(x_{0}\right)\right|<\varepsilon / 2+\varepsilon / 4+\varepsilon / 4=\varepsilon$.

(3): Use the fact that $\|u \psi-\psi\|=\sup _{v \in B_{V}}|\langle v, u \psi\rangle-\langle v, \psi\rangle|$ and $B_{V}$ is $G$-invariant.

THEOREM 9.8. The following conditions are equivalent:

(1) $f \in S U C(G)$.

(2) $f=m_{v, \psi}$ for some continuous Banach co-representation $h: G \rightarrow$ $\operatorname{Iso}(V), v \in V$ and $\psi \in V^{*}$, with the property that $\varphi$ is norm $G$ continuous for every $\varphi$ in the weak* closure $\operatorname{cl}_{w^{*}}(G \psi)$.

Moreover, one can assume in (2) that $\mathrm{cl}_{w^{*}}(G \psi)$ separates points of $V$.

Proof. $(2) \Rightarrow(1)$ : Let $h: G \rightarrow \operatorname{Iso}(V)$ be a continuous co-homomorphism such that $f$ is a matrix coefficient of $h$. That is, we can choose $v \in V$ and $\psi \in V^{*}$ such that $f(g)=\langle v g, \psi\rangle=\langle v, g \psi\rangle$ for every $g \in G$. One can assume that $\|\psi\|=1$. The strong continuity of $h$ ensures that the dual restricted (left) action of $G$ on the weak ${ }^{*}$ compact unit ball $\left(V_{1}^{*}, w^{*}\right)$ is jointly continuous. Consider the orbit closure $X:=\operatorname{cl}_{w^{*}}(G \psi)$ in the compact $G$-space $\left(V_{1}^{*}, w^{*}\right)$. Define the continuous function $\widehat{v}: X \rightarrow \mathbb{R}$ induced by the vector $v$. Precisely, $\widehat{v}(x)=\langle v, x\rangle$ and in particular, $f(g)=\widehat{v}(g \psi)$. So $f$ comes from $X$ and the compactification $\nu: G \rightarrow X, g \mapsto g \psi$. It suffices to show that 
the $G$-system $X$ is SUC. Let $x_{0}$ be an arbitrary point in $X$ and let $w$ be an arbitrary vector in $V$. By the definition of the weak* topology and the corresponding uniformity on the compact space $X$ it suffices to show that for every $\varepsilon>0$ there exists a neighborhood $U(e)$ such that $\left|\widehat{w}\left(g u x_{0}\right)-\widehat{w}\left(g x_{0}\right)\right| \leq \varepsilon$ for all $g \in G$ and $u \in U$. By simple computations we get

$$
\begin{aligned}
\left|\widehat{w}\left(g u x_{0}\right)-\widehat{w}\left(g x_{0}\right)\right| & =\left|\left\langle w, g u x_{0}\right\rangle-\left\langle w, g x_{0}\right\rangle\right|=\left|\left\langle w g, u x_{0}\right\rangle-\left\langle w g, x_{0}\right\rangle\right| \\
& =\left|\left\langle w g, u x_{0}-x_{0}\right\rangle\right| \leq\|w g\| \cdot\left\|u x_{0}-x_{0}\right\| .
\end{aligned}
$$

Take into account that $\|w g\|=\|w\|$. Since $x_{0} \in \operatorname{cl}(G \psi)$, by our assumption the orbit map $\widetilde{x}_{0}: G \rightarrow V^{*}$ is norm continuous with respect to the dual action of $G$ on $V^{*}$. Therefore, given $\varepsilon>0$, there exists a neighborhood $U$ of $e$ in $G$ such that $\left\|u x_{0}-x_{0}\right\|<\|w\|^{-1} \varepsilon$ for every $u \in U$. Thus, $\left|\widehat{w}\left(g u x_{0}\right)-\widehat{w}\left(g x_{0}\right)\right| \leq \varepsilon$. This shows that $X$ is SUC. Hence, $f \in S U C(G)$.

$(1) \Rightarrow(2)$ : Let $f \in S U C(X)$. Then by Proposition 3.1(3) it comes from a compact transitive $G$-system $X_{f}$ and the $G$-compactification $f_{\sharp}: G \rightarrow X_{f}$. There exists $F:=F_{e} \in C\left(X_{f}\right)$ such that $f=F \circ f_{\sharp}$. By Lemma 9.6(2) we conclude that $F G$ is SUC-small for $X_{f} \subset C\left(X_{f}\right)^{*}$.

Let $M:=\operatorname{co}(-F G \cup F G)$ be the convex hull of the symmetric set $-F G \cup$ $F G$. Then $M$ is a convex symmetric bounded $G$-invariant subset in $C\left(X_{f}\right)$. By Lemma 9.7(1) we know that $M$ is also SUC-small for $X_{f}$.

For brevity let $E$ denote the Banach space $C\left(X_{f}\right)$. Since $X_{f}$ is a compact $G$-space the natural right action of $G$ on $E=C\left(X_{f}\right)$ (by linear isometries) is continuous.

Consider the sequence $K_{n}:=2^{n} M+2^{-n} B_{E}$, where $B_{E}$ is the unit ball of $E$. Since $M$ is convex and symmetric, we can apply the construction of [7] (we mostly use the presentation and the development given by Fabian in the book [10]). Let \|\|$_{n}$ be the Minkowski functional of the set $K_{n}$. That is,

$$
\|v\|_{n}=\inf \left\{\lambda>0 \mid v \in \lambda K_{n}\right\}
$$

Then \|\|$_{n}$ is a norm on $E$ equivalent to the given norm of $E$ for every $n \in \mathbb{N}$. For $v \in E$, let

$$
N(v):=\left(\sum_{n=1}^{\infty}\|v\|_{n}^{2}\right)^{1 / 2} \text { and } \quad V:=\{v \in E \mid N(v)<\infty\}
$$

Denote by $j: V \hookrightarrow E$ the inclusion map. Then $(V, N)$ is a Banach space, $j: V \rightarrow E$ is a continuous linear injection and

$$
M \subset j\left(B_{V}\right)=B_{V} .
$$

Indeed, if $v \in M$ then $2^{n} v \in K_{n}$. Therefore, $\|v\|_{n} \leq 2^{-n}$ and $N(v)^{2} \leq$ $\sum_{n \in \mathbb{N}} 2^{-2 n}<1$.

By our construction, $M$ and $B_{E}$ are $G$-invariant. This implies that the natural right action $V \times G \rightarrow V,(v, g) \mapsto v g$, is isometric, that is, $N(v g)=$ 
$N(v)$. Moreover, by the definition of the norm $N$ on $V$ (use the fact that the norm $\|\cdot\|_{n}$ on $E$ is equivalent to the given norm of $E$ for each $n \in \mathbb{N}$ ) we can show that this action is norm continuous. Therefore, the co-representation $h: G \rightarrow \operatorname{Iso}(V), h(g)(v):=v g$, on the Banach space $(V, N)$ is well defined and continuous.

Let $j^{*}: E^{*} \rightarrow V^{*}$ be the adjoint map of $j: V \rightarrow E$. Now our aim is to check the $G$-continuity of every vector $\varphi \in j^{*}\left(X_{f}\right)=\operatorname{cl}_{w^{*}}(G \psi)$, where $\psi:=j^{*}(z)$ and $z$ denotes the point $f_{\sharp}(e) \in X_{f}$. By Lemma 9.7(3) we have to show that $B_{V}$ is SUC-small for $j^{*}\left(X_{f}\right)$.

Claim. $j\left(B_{V}\right) \subset \bigcap_{n \in \mathbb{N}} K_{n}=\bigcap_{n \in \mathbb{N}}\left(2^{n} M+2^{-n} B_{E}\right)$.

Proof. The norms $\|\cdot\|_{n}$ on $E$ are equivalent to each other. It follows that if $v \in B_{V}$ then $\|v\|_{n}<1$ for all $n \in \mathbb{N}$. That is, $v \in \lambda_{n} K_{n}$ for some $0<\lambda_{n}<1$ and $n \in \mathbb{N}$. By the construction, $K_{n}$ is a convex subset containing the origin. This implies that $\lambda_{n} K_{n} \subset K_{n}$. Hence $j(v)=v \in K_{n}$ for every $n \in \mathbb{N}$.

Recall now that $F G$ is SUC-small for $X_{f} \subset C\left(X_{f}\right)^{*}$. By Lemma 9.7(1) we know that also $M:=\operatorname{co}(-F G \cup F G)$ is SUC-small for $X_{f} \subset C\left(X_{f}\right)^{*}$. Moreover by Lemma 9.7(2) we find that $A:=\bigcap_{n \in \mathbb{N}}\left(2^{n} M+2^{-n} B_{E}\right) \subset C\left(X_{f}\right)$ is SUC-small for $X_{f} \subset C\left(X_{f}\right)^{*}$. The linear continuous operator $j: V \rightarrow$ $C\left(X_{f}\right)$ is a $G$-map. Then by Lemma 9.6(1) it follows that $j^{-1}(A) \subset V$ is SUC-small for $j^{*}\left(X_{f}\right) \subset V^{*}$. The same is true for $B_{V}$ because by the above claim we have $j\left(B_{V}\right) \subset A$ (and hence $B_{V} \subset j^{-1}(A)$ ). That is, $B_{V}$ is SUCsmall for $j^{*}\left(X_{f}\right)$. Now Lemma 9.7(3) shows that the orbit map $\widetilde{\varphi}: G \rightarrow V^{*}$ is $G$-continuous for every $\varphi \in j^{*}\left(X_{f}\right)=\mathrm{cl}_{w^{*}}(G \psi)$. By our construction, $F \in j(V)$ (because $F \in M \subset j\left(B_{V}\right)$ ). Since $j$ is injective, the element $v:=j^{-1}(F)$ is uniquely determined in $V$. We show that $f=m_{v, \psi}$ for the co-representation $h$. Using the equality $F \circ \alpha_{f}=f$ and the fact that $\alpha_{f}$ is a $G$-map we get

$$
\langle F g, z\rangle=F\left(g \alpha_{f}(e)\right)=\left(F \circ \alpha_{f}\right)(g)=f(g) .
$$

On the other hand,

$$
\left.m_{v, \psi}(g)=\langle v g, \psi\rangle=\left\langle j^{-1}(F) g, j^{*}(z)\right\rangle=\left\langle j\left(j^{-1}(F)\right) g\right), z\right\rangle=\langle F g, z\rangle .
$$

Hence, $f=m_{v, \psi}$, as required. Thus we have proved that $(1) \Leftrightarrow(2)$.

Finally, we show that one can assume in (2) that $\operatorname{cl}_{w^{*}}(G \psi)=j^{*}\left(X_{f}\right)$ separates points of $V$. If $v_{1}, v_{2}$ are different elements in $V$ then $j\left(v_{1}\right) \neq j\left(v_{2}\right)$. Since $X_{f}$ separates $C\left(X_{f}\right)$ we have $\left\langle j\left(v_{1}\right), \phi\right\rangle \neq\left\langle j\left(v_{2}\right), \phi\right\rangle$ for some $\phi \in X_{f}$. Now observe that $\langle j(v), \phi\rangle=\left\langle v, j^{*}(\phi)\right\rangle$ for every $v \in V$.

Corollary 9.9. $\mathcal{A} d j(G) \subset S U C(G)$.

Next we show how one can characterize $L E(G)$ in terms of matrix coefficients. 
THEOREM 9.10. The following conditions are equivalent:

(1) $f \in L E(G)$.

(2) $f=m_{v, \psi}$ for some continuous co-representation $h: G \rightarrow \operatorname{Iso}(V)$, $v \in V$ and $\psi \in V^{*}$, for a Banach space $V$, with the property that the weak $^{*}$ and the norm topologies coincide on the orbit $G \varphi$ of every $\varphi$ in the weak $k^{*}$ closure $Y:=\mathrm{cl}_{w^{*}}(G \psi)$.

Moreover, one can assume in (2) that $Y$ separates points of $V$.

Proof. $(2) \Rightarrow(1)$ : By definition $f$ comes from $Y:=\operatorname{cl}_{w^{*}}(G \psi)$. Hence it suffices to show that $Y$ is LE. Equivalently, we need to show that $Y$ is orbitwise light (see [13, Lemma 5.8(2)]). Let $\mu_{Y}$ be the uniform structure on the compact space $Y$. Denote by $\left(\mu_{Y}\right)_{G}$ the corresponding uniform structure of uniform convergence inherited from $Y^{G}$ (see [13]). We have to show that $\left.\operatorname{top}\left(\mu_{Y}\right)\right|_{G \varphi}=\left.\operatorname{top}\left(\left(\mu_{Y}\right)_{G}\right)\right|_{G \varphi}$ for every $\varphi \in Y$. Observe that the topology $\left(\mu_{Y}\right)_{G}$ on the orbit $G \varphi$ is weaker than the norm topology. Since the latter is the same as the weak ${ }^{*}$ topology (that is, $\left.\operatorname{top}\left(\mu_{Y}\right)\right|_{G \varphi}$ ) we see that indeed $\left.\operatorname{top}\left(\mu_{Y}\right)\right|_{G \varphi}=\left.\operatorname{top}\left(\left(\mu_{Y}\right)_{G}\right)\right|_{G \varphi}$.

$(1) \Rightarrow(2)$ : The proof uses again the interpolation technique of [7], as in Theorem 9.8. The proof is similar so we omit the details. However, we provide necessary definition and two lemmas (Definition 9.11 and Lemmas 9.12 and 9.13). They play the role of Lemmas 9.6 and 9.7.

For every set $M$ denote by $\mathbb{R}^{M}$ the set of all real-valued functions $M \rightarrow \mathbb{R}$. The topologies of pointwise and uniform convergence on $\mathbb{R}^{M}$ will be denoted by $\tau_{p}$ and $\tau_{u}$ respectively.

Definition 9.11. Let $\langle\rangle:, Y \times X \rightarrow \mathbb{R}$ be a $G$-compatible map (as in Definition 9.5) and $M$ be a nonempty subset of $Y$. Denote by $j: X \rightarrow \mathbb{R}^{M}$, $j(x)(m):=\langle m, x\rangle$, the associated map.

(1) We say that a subset $A$ of $X$ is $M$-light if the pointwise and uniform topologies coincide on $j(A) \subset \mathbb{R}^{M}$.

(2) $M$ is $L E$-small at $x_{0} \in X$ if the orbit $G x_{0}$ is $M$-light.

(3) $M$ is $L E$-small for $X$ if the orbit $G x$ is $M$-light at every $x \in X$ (cf. Theorem 7.9).

We are going to examine this definition in a particular case of the canonical bilinear map $V \times V^{*} \rightarrow \mathbb{R}$ which is $G$-compatible for every co-representation $h: G \rightarrow \operatorname{Iso}(V)$.

We collect here some useful properties of LE-smallness.

LEMMA 9.12.

(1) Let $Y_{1} \times X_{1} \rightarrow \mathbb{R}$ and $Y_{2} \times X_{2} \rightarrow \mathbb{R}$ be two $G$-compatible maps. Suppose that $\gamma_{1}: X_{1} \rightarrow X_{2}$ and $\gamma_{2}: Y_{2} \rightarrow Y_{1}$ are $G$-maps such that

$$
\left\langle y, \gamma_{1}(x)\right\rangle=\left\langle\gamma_{2}(y), x\right\rangle \quad \forall(y, x) \in Y_{2} \times X_{1} .
$$


Then for every nonempty subset $M \subset Y_{2}$ the subset $\gamma_{1}(M) \subset Y_{1}$ is $L E$-small at $x \in X_{1}$ if and only if $M$ is LE-small at $\gamma_{1}(x) \in X_{2}$.

(2) Let $X$ be a (not necessarily compact) $G$-space. If $f \in L E(X)$ then the subset $F_{e} G$ of $C\left(X_{f}\right)$ is LE-small for $X_{f}$ considered as a subset of $V^{*}$ where $V:=C\left(X_{f}\right)$ (with respect to the canonical map $V \times V^{*} \rightarrow \mathbb{R}$ and the natural co-representation $G \rightarrow \operatorname{Iso}(V))$.

Proof. (1): Similar to Lemma 9.6.1.

(2): Let $f \in L E(X)$. Then by Proposition 3.1.3 it comes from a compact $G$-system $X_{f}$ and the $G$-compactification $f_{\sharp}: X \rightarrow X_{f}$. we know that there exists $F_{e} \in C\left(X_{f}\right)$ such that $f=F_{e} \circ f_{\sharp}$. Theorem 7.9 implies that $X_{f}$ is LE and $F_{e} \in L E\left(X_{f}\right)$. By the same theorem, $X_{f}$ is orbitwise light in $R U C(G)$. This means that pointwise and norm topologies in $R U C(G)$ agree on every $G$-orbit in $X_{f}$. On the other hand, it is straightforward to see that for the $G$-compatible map

$$
F_{e} G \times X_{f} \rightarrow \mathbb{R}
$$

(Definition 9.11 with $M:=F_{e} G$ ) the corresponding pointwise topology $\tau_{p}$ on $X_{f}$ coincides with the pointwise topology inherited from $R U C(G)$, and the uniform topology $\tau_{u}$ on $X_{f}$ coincides with the norm topology of $R U C(G)$.

Lemma 9.13. Let $h: G \rightarrow \operatorname{Iso}(V)$ be a continuous co-representation.

(a) For every $X \subset V^{*}$ the family of LE-small sets for $X$ in $V$ is closed under taking: subsets, norm closures, finite linear combinations, finite unions and convex hulls.

(b) If $M_{n} \subset V$ is LE-small at $x_{0} \in V^{*}$ for every $n \in \mathbb{N}$ then so is $\bigcap_{n \in \mathbb{N}}\left(M_{n}+\delta_{n} B_{V}\right)$ for every positive decreasing sequence $\delta_{n} \rightarrow 0$.

(c) The following are equivalent:

(i) The pointwise and norm topologies agree on the G-orbit Gx for every $x \in X \subset V^{*}$.

(ii) $\mathcal{B}$ is LE-small for $X$, where $\mathcal{B}:=\left\{\check{v}: V^{*} \rightarrow \mathbb{R}, x \mapsto \check{v}(x):=\right.$ $\langle v, x\rangle\}_{v \in B_{V}}$.

Question 9.14. Do we have adj $(G)=S U C(G)$ for every topological group $G$ ? The question seems to be open even for abelian non-discrete $G$ (say $G=\mathbb{R})$. The equivalent question for abelian $G$ is whether $\operatorname{adj}(G)=U C(G)$. Also, what is the relation between the algebras $L E(G)$ and $\operatorname{adj}(G)$ ?

10. Some conclusions about $H_{+}[0,1]$ and $\operatorname{Iso}\left(\mathbb{U}_{1}\right)$. From the reflexive triviality of $H_{+}[0,1]$ and results of Uspenskij about Iso $\left(\mathbb{U}_{1}\right)$ Pestov deduces in $\left[39\right.$, Corollary 1.4] the fact that the group Iso $\left(\mathbb{U}_{1}\right)$ is also reflexively trivial. Using a similar idea and the matrix coefficient characterization of SUC and LE one can conclude that Iso $\left(\mathbb{U}_{1}\right)$ is SUC-trivial and LE-trivial.

Recall the following results of Uspenskij. 
TheOREM 10.1 (Uspenskij $[45,47])$. The group $\operatorname{Iso}\left(\mathbb{U}_{1}\right)$ is topologically simple and contains a copy of every second countable topological group (e.g., $\left.H_{+}[0,1]\right)$.

Lemma 10.2 below is a generalized version of Pestov's observation. Of course it is important here that the corresponding property admits a reformulation in terms of Banach space representations, which is the case, for instance, for SUC and LE.

Lemma 10.2. Let $G_{1}$ be a topological subgroup of a group $G_{2}$. Suppose that $G_{2}$ is $G_{1}$-simple, in the sense that, every nontrivial normal subgroup $N$ in $G_{2}$ containing $G_{1}$ is necessarily dense in $G_{2}$. Then if $G_{1}$ is either: (1) SUC-trivial, (2) LE-trivial, (3) adjoint continuous trivial or (4) $\mathcal{K}$-trivial (where $\mathcal{K}$ is a class of Banach spaces) then the same is true for $G_{2}$.

Proof. We consider only the case of SUC. Other cases are similar (and even easier for (3) and (4)).

We use Theorem 9.8. Let $h: G_{2} \rightarrow \operatorname{Iso}(V)$ be a continuous co-representation where $Y:=\operatorname{cl}_{w^{*}}(G \psi)$ separates points of $V$ and $\varphi$ is norm $G_{2}$-continuous in $V^{*}$ for every $\varphi \in Y$. It is enough to show that any such co-representation of $G_{2}$ is trivial. By Theorem 9.8 this will show that $G_{2}$ is SUC-trivial. First observe that the restriction $\left.h\right|_{G_{1}}$ is trivial. In fact, otherwise $v g \neq v$ for some $(v, g) \in V \times G_{1}$ and by our assumption there exists $\varphi \in Y$ such that $\varphi(v) \neq \varphi(v g)$. Then the restriction $\left.m_{v, \varphi}\right|_{G_{1}}$ of the corresponding matrix coefficient $m_{v, \varphi}: G_{2} \rightarrow \mathbb{R}$ to $G_{1}$ is not constant. However, by Theorem 9.8, $\left.m_{v, \varphi}\right|_{G_{1}} \in S U C\left(G_{1}\right)$, contradicting our assumption that $G_{1}$ is SUC-trivial. Therefore, $\left.h\right|_{G_{1}}: G_{1} \rightarrow \operatorname{Iso}(V)$ is trivial. Hence $G_{1}$ is a subgroup of the normal closed subgroup $N:=\operatorname{ker}(h)$ of $G_{2}$. Since $G_{2}$ is $G_{1}$-simple it follows that $N=G_{2}$. Hence $h$ is trivial.

Note that if $G_{2}$ is topologically simple, that is, $\{e\}$-simple, then it is $G_{1}$-simple for every subgroup.

The following theorem sums up some of our results concerning the topological groups $H_{+}[0,1]$ and $\operatorname{Iso}\left(\mathbb{U}_{1}\right)$.

TheOREM 10.3. Let $G$ be one of the groups $H_{+}[0,1]$ or $\operatorname{Iso}\left(\mathbb{U}_{1}\right)$.

(1) The compactifications $G^{S U C}, G^{L E}, G^{\text {Asp }}, G^{W A P}$ are trivial.

(2) Every adjoint continuous (co)representation of the group $G$ is trivial.

(3) Every continuous Asplund (co)representation of the group $G$ is trivial.

(4) Every (co)representation $h: G \rightarrow \operatorname{Iso}(V)$ on a separable Asplund space $V$ is trivial.

(5) The algebra $U C(G)$ and the ambit $\left(G^{U C}, i(e)\right)$ are not point-universal. In particular, the map $i: G \rightarrow G^{U C}$ is not a right topological compactification of $G$. 
Proof. (1): $H_{+}[0,1]$ is SUC-trivial by Theorem 8.3. By results of Uspenskij (see Theorem 10.1) the group Iso( $\left.\mathbb{U}_{1}\right)$ is topologically simple and also a universal second countable group. In particular it contains a copy $G_{1}$ of $H_{+}[0,1]$ as a topological subgroup. It follows that $G_{2}:=\operatorname{Iso}\left(\mathbb{U}_{1}\right)$ is $G_{1}$-simple. Applying Lemma 10.2 we conclude that $G_{2}:=\operatorname{Iso}\left(\mathbb{U}_{1}\right)$ is also SUC-trivial. The rest follows by the inclusions of Theorem 7.10.

(2): Every adjoint continuous (co)representation of $H_{+}[0,1]$ must be trivial. Otherwise, by Theorem 9.8 (or Corollary 9.9), it contains a nonconstant SUC function. Now Lemma 10.2 implies that Iso $\left(\mathbb{U}_{1}\right)$ is also adjoint continuous trivial.

(3): By Theorem 6.5 every continuous Asplund (co)representation of $G$ is adjoint continuous. Now apply (2).

(4): By a recent result of Rosendal and Solecki [42, Corollary 3] every homomorphism of $G=H_{+}[0,1]$ into a separable group is necessarily continuous. Combining this result and our assertion (3) we obtain the proof in the case of $G=H_{+}[0,1]$. The case of $G=\operatorname{Iso}\left(\mathbb{U}_{1}\right)$ now follows by using again the $H_{+}[0,1]$-simplicity of $\operatorname{Iso}\left(\mathbb{U}_{1}\right)$.

(5): Take a nonconstant uniformly continuous function on $G$ (such a function necessarily exists by Lemma 2.1 ). Since $S U C(G)=\{$ constants $\}$ we get $S U C(G) \neq U C(G)$. Now Corollary 4.11 finishes the proof.

By Theorems 10.1 and 10.3 we get

COROLlary 10.4. Every second countable group $G_{1}$ is a subgroup of a Polish SUC-trivial group $G_{2}$.

However the following questions are open (see also [31]).

QUESTION 10.5.

(1) Find a nontrivial Polish group which is SUC-trivial (Ref-trivial, $\mathcal{A}$ sptrivial) but does not contain a subgroup topologically isomorphic to $H_{+}[0,1]$.

(2) Is the group $H\left(I^{\omega}\right)$ SUC-trivial (Ref-trivial, $\mathcal{A} s p$-trivial)? And, a closely related question (see Lemma 10.2):

(3) Is the group $G_{2}:=H\left(I^{\omega}\right) G_{1}$-simple for a subgroup $G_{1}<G_{2}$ where $G_{1}$ is a copy of either $H_{+}[0,1]$ or $\operatorname{Iso}\left(\mathbb{U}_{1}\right)$ ?

Theorem 10.6. Let $G$ be an Asplund trivial (e.g. $H_{+}[0,1]$ or $\operatorname{Iso}\left(\mathbb{U}_{1}\right)$ ) group. Then every metrizable right topological semigroup compactification of $G$ is trivial.

Proof. By Theorem 10.3.1, $G^{\text {Asp }}$ is trivial, so that every RN transitive $G$-space is trivial. If $G \rightarrow S$ is a right topological semigroup compactification of $G$, then the natural induced $G$-space $(G, S)$ is isomorphic to its own enveloping semigroup. By a recent work [14], a metric dynamical system 
$(G, X)$ is RN iff its enveloping semigroup is metrizable. Now if $S$ is metrizable then it follows that the transitive system $(G, S)$ is $\mathrm{RN}$ and therefore trivial.

Recall, in contrast, that for every topological group $G$ the algebra $R U C(G)$ separates points and closed subsets on $G$ and therefore the maximal right topological semigroup compactification $G \hookrightarrow G^{R U C}$ is faithful.

11. Relative extreme amenability: SUC-fpp groups. Recall that a topological group $G$ has the fixed point property on compacta (fpp) (or is extremely amenable) if every compact (transitive) $G$-space $X$ has a fixed point. It is well known that locally compact extremely amenable groups are necessarily trivial (see for example [19]). Gromov and Milman [20] proved that the unitary group $U(H)$ is extremely amenable. Pestov has shown that the groups $H_{+}[0,1]$ and Iso $\left(\mathbb{U}_{1}\right)$ are extremely amenable (see $[37,40]$ for more information).

Consider the following relativization.

Definition 11.1. Let $\mathrm{P}$ be a class of compact $G$-spaces.

(1) A $G$-space $X$ is $P$-fpp (or is extremely $P$-amenable) if every $G$-compactification $X \rightarrow Y$ such that $Y$ is a member of $\mathrm{P}$ has a fixed point.

(2) A topological group $G$ is $P$-fpp (or is extremely P-amenable) if the $G$-space $X:=G$ is P-fpp or equivalently, if every $G$-space $Y$ in $\mathrm{P}$ has a fixed point.

Taking $\mathrm{P}$ as the collection of all compact flows we get extreme amenability. With the class $\mathrm{P}$ of compact affine flows we recover amenability. When $\mathrm{P}$ is taken to be the collection of equicontinuous (that is, almost periodic) flows we obtain the old notion of minimal almost periodicity (MAP). Minimal almost periodicity was first studied by von Neumann and Wigner [34] who showed that $\operatorname{PSL}(2, \mathbb{Q})$ has this property. See also Mitchell [33] and Berglund, Junghenn and Milnes [5].

Lemma 11.2. Let $P$ be a class of compact $G$-spaces which is preserved by isomorphisms, products subsystems and quotients. Let $\mathcal{P}$ and $X^{\mathcal{P}}$ be as in Section 2. The following conditions are equivalent:

(1) $G$ is P-fpp.

(2) The compact $G$-space $G^{\mathcal{P}}$ is P-fpp.

(3) Any minimal compact $G$-space in $P$ is trivial.

(4) For every $f \in \mathcal{P}$ the $G$-system $X_{f}$ has a fixed point.

(5) The algebra $\mathcal{P}$ is extremely left amenable (that is, it admits a multiplicative left invariant mean). 
Proof. Clearly each of the conditions (1) and (3) implies all the others. Use the fact that the $G$-space $G^{\mathcal{P}}$ is point universal to deduce that each of (2) and (5) implies (3). Finally, (4) implies (2) because $G^{\mathcal{P}}$ has a presentation as a subsystem of the product of all the $X_{f}, f \in \mathcal{P}$. Note that $f \in \mathcal{P}$ iff $X_{f}$ has property $\mathrm{P}$ (see [13, Proposition 2.9.3]).

REMARK 11.3.

(1) The smaller the class $\mathrm{P}$ is, one expects the property of being P-fpp to be less restrictive; however, even when one takes $\mathrm{P}$ to be the class of equicontinuous $\mathbb{Z}$-spaces (that is, cascades) it is still an open question whether P-fpp, that is, minimal almost periodicity, is equivalent to extreme amenability (see [11]).

(2) A minimal compact $G$-space $X$ is LE iff $X$ is AP. It follows by the inclusions $\mathrm{LE} \supset \mathrm{RN}_{\text {app }} \supset \mathrm{WAP} \supset \mathrm{AP}$ (cf. also Theorem 7.10) that $G$ is minimally almost periodic iff $G$ is P-fpp for each of the following classes: WAP, $\mathrm{RN}_{\text {app }}$ or LE.

Here we point out two examples of topological groups $G$ which are SUCextremely amenable (equivalently, SUC-fpp) but not extremely amenable. In the next two sections we will show that $S_{\infty}$ as well as the group $H(C)$ of homeomorphisms of the Cantor set $C$ are also SUC-fpp (both groups are not extremely amenable). See Corollary 12.3 and Theorem 13.8 below.

EXAmple 11.4. For every $n \geq 2$ the simple Lie group $S L_{n}(\mathbb{R})$, being locally compact, is not extremely amenable. However, it is SUC-extremely amenable. This follows easily from Corollary 7.15.

For the second example we need the following proposition.

Proposition 11.5. Let $G$ be a uniformly Lindelöf (e.g. second countable) group and $X$ a not necessarily compact $G$-space. Assume that the action is 2-homogeneous on some uncountable dense orbit $Y=G x_{0}$. Then $X$ is SUC-trivial (and hence also SUC-fpp).

Proof. It is enough to show that every $f \in S U C(X)$ is constant. We can suppose that $Y=X=G x_{0}$. Let $\operatorname{St}\left(x_{0}\right)<G$ be the stabilizer of $x_{0}$. We observe that $\operatorname{St}\left(x_{0}\right)$ is not open in $G$. Indeed, otherwise the space $G / \operatorname{St}\left(x_{0}\right)$ is discrete and since $G$ is uniformly Lindelöf it is easy to see that $G / \operatorname{St}\left(x_{0}\right)$ is countable. However, by assumption $X$ is uncountable and the cardinality of $X$ is the same as that of the coset space $G / \operatorname{St}\left(x_{0}\right)$. It follows that $U x_{0} \neq\left\{x_{0}\right\}$ for every neighborhood $U$ of the identity $e \in G$. Now suppose to the contrary that $f$ is a nonconstant SUC function and let $\varepsilon:=|f(a)-f(b)|>0$ for some $a, b \in X$. By the definition of SUC there exists a neighborhood $U(e)$ such that

$$
\left|f\left(g u x_{0}\right)-f\left(g x_{0}\right)\right|<\varepsilon \quad \forall(g, u) \in G \times U .
$$


As was already established, there exists $x_{1}=u_{0} x_{0} \in U x_{0}$ such that $x_{1} \neq x_{0}$. Since the action is 2-homogeneous we can choose $g_{0} \in G$ such that $g_{0} x_{1}=a$ and $g_{0} x_{0}=b$. Then $\left|f\left(g_{0} u_{0} x_{0}\right)-f\left(g_{0} x_{0}\right)\right|=\varepsilon$, a contradiction.

Now for many concrete homogeneous uncountable metric compact spaces $X$ the natural action of the topological group $G=H(X)$ on $X$ is 2homogeneous. By Proposition 11.5 the flow $(G, X)$ admits only constant SUC functions and the corresponding SUC $G$-compactification $X^{S U C}$ is trivial. This is the case, to mention some concrete examples, for $X$ the Cantor set (see Remark 13.5), the Hilbert cube and the circle $\mathbb{T}$.

In the latter case even the subgroup $G:=H_{+}(\mathbb{T})<H(\mathbb{T})$ of all orientationpreserving homeomorphisms of the circle acts 2 -homogeneously on $\mathbb{T}$. Pestov has shown $[37,40]$ that the universal minimal dynamical $G$-system $M(G)$ for $G:=H_{+}(\mathbb{T})$ coincides with the natural action of $G$ on $\mathbb{T}$. Combining these results with Proposition 11.5 we obtain our second example.

Corollary 11.6. The Polish group $G=H_{+}(\mathbb{T})$ of orientation preserving homeomorphisms of the circle is SUC-extremely amenable (but it is not extremely amenable).

An alternative proof follows easily from Proposition 5.1.

12. The Roelcke compactification of the group $S(\mathbb{N})$. Let $G=$ $S(\mathbb{N})$ be the Polish topological group of all permutations of the set $\mathbb{N}$ of natural numbers (equipped with the topology of pointwise convergence). Consider the one-point compactification $X^{*}=\mathbb{N} \cup\{\infty\}$ and the associated natural $G$-action $\left(G, X^{*}\right)$. For any subset $A \subset \mathbb{N}$ and an injection $\alpha: A \rightarrow \mathbb{N}$ let $p_{\alpha}$ be the map in $\left(X^{*}\right)^{X^{*}}$ defined by

$$
p_{\alpha}(x)= \begin{cases}\alpha(x), & x \in A, \\ \infty, & \text { otherwise. }\end{cases}
$$

We have the following simple claim.

Claim 12.1. The enveloping semigroup $E=E\left(G, X^{*}\right)$ of the $G$-system $\left(G, X^{*}\right)$ consists of the maps $\left\{p_{\alpha}: \alpha: A \rightarrow \mathbb{N}\right\}$ as above. Every element of $E$ is a continuous function so that by the Grothendieck-Ellis-Nerurkar theorem [9], the system $\left(G, X^{*}\right)$ is WAP.

Proof. Let $\pi_{\nu}$ be a net of elements of $S(\mathbb{N})$ with $p=\lim _{\nu} \pi_{\nu}$ in $E$. Let $A=\{n \in \mathbb{N}: p(n) \neq \infty\}$ and $\alpha(n)=p(n)$ for $n \in A$. Clearly $\alpha: A \rightarrow \mathbb{N}$ is an injection and $p=p_{\alpha}$.

Conversely, given $A \subset \mathbb{N}$ and an injection $\alpha: A \rightarrow \mathbb{N}$ we construct a sequence $\pi_{n}$ of elements of $S(\mathbb{N})$ as follows. Let $A_{n}=A \cap[1, n]$ and 
$M_{n}=\max \left\{\alpha(i): i \in A_{n}\right\}$. Next define an injection $\beta_{n}:[1, n] \rightarrow \mathbb{N}$ by

$$
\beta_{n}(j)= \begin{cases}\alpha(j), & j \in A, \\ j+M_{n}+n, & \text { otherwise. }\end{cases}
$$

Extending the injection $\beta_{n}$ to a permutation $\pi_{n}$ of $\mathbb{N}$ in an arbitrary way, we now observe that $p_{\alpha}=\lim _{n \rightarrow \infty} \pi_{n}$ in $E$. The last assertion is easily verified.

THEOREM 12.2 .

(1) The two algebras $U C(G)$ and $W A P(G)$ coincide.

(2) The universal WAP compactification $G^{W A P}$ of $G$ (and hence also $G^{U C}$ ) is isomorphic to $E=E\left(G, X^{*}\right)$. Thus the universal WAP (and Roelcke) compactification of $G$ is homeomorphic to the Cantor set.

Proof. Given $f \in U C(G)$ and an $\varepsilon>0$ there exists $k \in \mathbb{N}$ such that, with $H=H(1, \ldots, k)=\{g \in G: g(j)=j, \forall 1 \leq j \leq k\}$,

$$
\sup _{u, v \in H}|f(u g v)-f(g)|<\varepsilon \text {. }
$$

Set $\widehat{f}(g)=\sup _{u, v \in H} f(u g v)$. Then $\|\widehat{f}-f\| \leq \varepsilon$. Clearly $\widehat{f}$, being $H$-biinvariant, is both right and left uniformly continuous, i.e. $\widehat{f} \in U C(G)$. Let

$$
\mathbb{N}_{*}^{k}=\left\{\left(n_{1}, \ldots, n_{k}\right): n_{j} \in \mathbb{N} \text { are distinct }\right\}=\{\text { injections }\{1, \ldots, k\} \rightarrow \mathbb{N}\}
$$

and let $G$ act on $\mathbb{N}_{*}^{k}$ by

$$
g\left(n_{1}, \ldots, n_{k}\right)=\left(g^{-1} n_{1}, \ldots, g^{-1} n_{k}\right) .
$$

The stability group of the point $(1, \ldots, k) \in \mathbb{N}_{*}^{k}$ is just $H$ and we can identify the discrete $G$-space $G / H$ with $\mathbb{N}_{*}^{k}$. Under this identification, to a function $f \in U C(G)$ which is right $H$-invariant (that is, $f(g h)=f(g)$ for all $g \in G$ and $h \in H$ ) corresponds a bounded function $\omega_{f} \in \Omega_{k}=\mathbb{R}^{\mathbb{N}_{*}^{k}}$, namely

$$
\omega_{f}\left(n_{1}, \ldots, n_{k}\right)=f(g) \quad \text { iff } \quad g(j)=n_{j}, \forall 1 \leq j \leq k .
$$

If we now assume that $f \in U C(G)$ is both right and left $H$-invariant (so that $f=\widehat{f}$ ) then, as we will see below, $f$ and accordingly its corresponding $\omega_{f}$ admit only finitely many values, corresponding to the finitely many double $H$-cosets $\{H g H: g \in G\}$.

We set $Y_{f}=Y=\operatorname{cl}\left\{g \omega_{f}: g \in G\right\} \subset \Omega_{k}=\mathbb{R}^{\mathbb{N}_{*}^{k}}$, where the closure is with respect to the pointwise convergence topology. Then $\left(G, Y_{f}\right)$ is a compact $G$-system which is isomorphic, via the identification $G / H \cong \mathbb{N}_{*}^{k}$, to $X_{f} \subset \mathbb{R}^{G}$. We will refer to elements of $\Omega_{k}=\mathbb{R}^{\mathbb{N}_{*}^{k}}$ as configurations. Consider first the case $k=2$.

In the following figure we have a representation of the configuration $f=$ $\omega_{f}=\omega_{1,2}$ and three other typical elements of $Y_{f}$. The configuration $\omega_{2,7}=$ $\sigma \omega_{1,2}$, where $\sigma$ is the permutation $\left(\begin{array}{lll}1 & 2 & 7 \\ 7 & 1 & 2\end{array}\right)$, admits seven values (the maximal number it can possibly have): "blank" at points $(m, n)$ with $m, n \notin\{2,7\}$, 
the values $\diamond$ and $*$ at $(2,7)$ and $(7,2)$ respectively, and four more constant values on the two horizontal and two vertical lines. (The circled diagonal points $(2,2)$ and $(7,7)$ are by definition not in $\mathbb{N}_{*}^{2}$.) If we let $\pi_{n}$ be the permutation

$$
\pi_{n}(j)= \begin{cases}j, & j \notin\{1, n\} \\ n, & \text { for } j=1, \\ 1, & \text { for } j=n,\end{cases}
$$

and denote by $p=\lim _{n \rightarrow \infty} \pi_{n}$ the corresponding element of $E(G, X)$, then e.g. $\omega_{1, \infty}=p \omega_{1,2}=\lim _{n \rightarrow \infty} \pi_{n} \omega_{1,2}=\lim _{n \rightarrow \infty} \omega_{1, n}$.
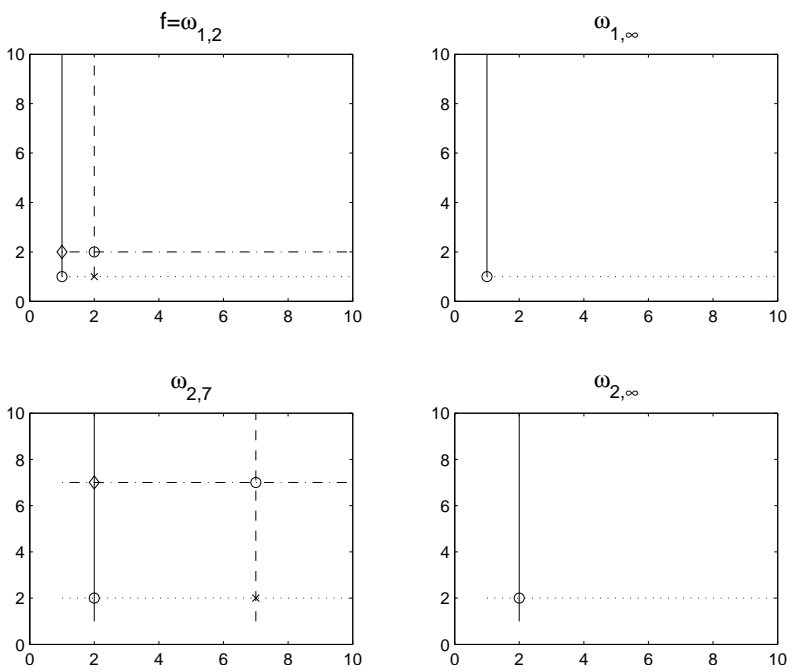

The functions $\omega_{1,2}, \omega_{1, \infty}, \omega_{2,7}$ and $\omega_{2, \infty}$ in $\Omega_{2}$

Now it is not hard to see that the $G$-action on $Y$ naturally extends to an action of $E=E\left(G, X^{*}\right)$ on $Y$ where each $p \in E$ acts continuously. (Show that the map $(a, b) \mapsto \omega_{a, b}$ is an isomorphism of $G$-systems from $X^{*} \times X^{*} \backslash \Delta$ onto $Y$, where $\Delta=\{(n, n): n \in \mathbb{N}\}$.) It then follows that $E=E\left(G, X^{*}\right)$ coincides with $E(G, Y)$.

By the Grothendieck-Ellis theorem (see e.g. [12, Theorem 1.45]) these observations show that the $G$-space $Y_{f}$ is WAP and therefore the function $f$, which comes from $\left(G, Y_{f}\right)$, is a WAP function.

These considerations are easily seen to hold for any positive integer $k$. For example, an easy calculation shows that for $H_{k}=H(1, \ldots, k)$, the number of double cosets $\left\{H_{k} g H_{k}: g \in G\right\}$ is

$$
\sum_{j=0}^{k}\left(\begin{array}{l}
k \\
j
\end{array}\right) \frac{k !}{(k-j) !} .
$$


Since the subgroups $H_{k}=H(1, \ldots, k)$ form a basis for the topology at $e$, as we have already seen, the union of the $H_{k}$-biinvariant functions for $k=$ $2,3, \ldots$ is dense in $U C(G)$ and we conclude that indeed $U C(G)=W A P(G)$.

Since for each $H$-biinvariant function $f$ the enveloping semigroup of the dynamical system $\left(G, Y_{f}\right)$ is isomorphic to $E\left(G, X^{*}\right)$ and since the Gelfand compactification of $U C(G)$ is isomorphic to a subsystem of the direct product

$$
\prod\left\{Y_{f}: f \text { is } H \text {-biinvariant for some } H(1, \ldots, k)\right\}
$$

we deduce that $E\left(G, X^{*}\right)$ also serves as the enveloping semigroup of the universal dynamical system $|W A P(G)|=|U C(G)|$. Finally, since $|W A P(G)|$ is point-universal we conclude (by Lemma 3.3) that $(G,|W A P(G)|)$ and $\left(G, E\left(G, X^{*}\right)\right)$ are $G$-isomorphic.

Corollary 12.3. The Polish group $G=S(\mathbb{N})$ is $S U C$-extremely amenable but not extremely amenable.

Proof. It was shown by Pestov [37] that $G$ is not extremely amenable and the nontrivial universal minimal $G$-system was described in [17]. On the other hand, the $G$-system $G^{U C}$ described in Theorem 12.2 admits a unique minimal set which is a fixed point. Thus the SUC $G$-system $G^{S U C}$, being $G$-isomorphic to $G^{U C}$ (see Theorems 12.2 and 7.10.2), has a fixed point.

13. The homeomorphisms group of the Cantor set. In this section let $C$ denote the classical Cantor set, i.e. the ternary subset of the interval $[0,1]$. Thus $C$ has the following representation:

$$
C=\bigcap_{n=0}^{\infty} I^{n},
$$

where $I^{n}=\bigcup_{j=1}^{2^{n}} I_{j}^{n}$ is the disjoint union of the $2^{n}$ closed intervals obtained by removing from $I=[0,1]$ the appropriate $2^{n}-1$ open "middle third" intervals. We will write $I_{j}^{m}$ for the clopen subset $I_{j}^{m} \cap C$ of $C$. For each integer $m \geq 1, \mathbf{I}^{m}=\left\{I_{j}^{m}: 1 \leq j \leq 2^{m}\right\}$ denotes the basic partition of $C$ into $2^{m}$ clopen "intervals".

We let $G=H(C)$ be the Polish group of homeomorphisms of $C$ equipped with the topology of uniform convergence. For $n \in \mathbb{N}$ we let

$$
H_{n}=\left\{g \in G: g I_{j}^{n}=I_{j}^{n}, \forall 1 \leq j \leq 2^{n}\right\} .
$$

Each $H_{n}$ is a clopen subgroup of $G$ and we note that the system of clopen subgroups $\left\{H_{n}: n=2,3, \ldots\right\}$ forms a basis for the topology of $G$ at the identity $e \in G$.

For any fixed integer $k \geq 1$ consider the collection $\mathcal{A}^{k}=\left\{\mathbf{a}=\left\{A_{1}, \ldots, A_{k}\right\}\right.$ : a partition of $C$ into $k$ nonempty clopen sets $\}$. In particular, for $k=2^{n}, \mathbf{I}^{n}$ is an element of $\mathcal{A}^{k}$. 
The discrete homogeneous space $G / H_{n}$ can be identified with $\mathcal{A}^{k}=\mathcal{A}^{2^{n}}$ : an element $g H_{n} \in G / H_{n}$ is uniquely determined by the partition

$$
\mathbf{a}=\left\{g I_{j}^{n}: 1 \leq j \leq 2^{n}=k\right\},
$$

and conversely to every partition $\mathbf{a} \in \mathcal{A}^{k}$ corresponds a coset $g H_{n} \in G / H_{n}$. In fact, if $\mathbf{a}=\left\{A_{1}, \ldots, A_{k}\right\}$ we can choose $g$ to be any homeomorphism of $C$ with $A_{j}=g I_{j}^{n}$.

Thus for $k=2^{n}$ we have a parametrization of $\mathcal{A}^{k}$ by the discrete homogeneous space $G / H_{n}$.

Let

$$
\Omega^{k}=\mathbb{R}^{\mathcal{A}^{k}} \cong \mathbb{R}^{G / H_{n}}
$$

Via the quotient map $G \rightarrow G / H_{n}, g \mapsto g H_{n}$, the Banach space $\ell^{\infty}\left(\mathcal{A}^{k}\right)$ canonically embeds into the Banach space $R U C(G)$ where the image consists of all the right $H_{n}$-invariant functions in $R U C(G)$. Thus if $f \in R U C(G)$ satisfies $f(g h)=f(g)$ for all $g \in G$ and $h \in H_{n}$ then $\omega_{f}\left(g I_{1}^{n}, \ldots, g I_{k}^{n}\right)=$ $\omega_{f}\left(A_{1}, \ldots, A_{k}\right)=f(g)$, where $A_{j}=g I_{j}^{n}$, is the corresponding configuration in $\ell^{\infty}\left(\mathcal{A}^{k}\right)$.

We equip $\Omega^{k}=\mathbb{R}^{\mathcal{A}^{k}}$ with its product topology. The group $G$ acts on the space $\Omega^{k}$ as follows. For $\omega \in \Omega^{k}$ and $g \in G$ let

$$
g \omega(\mathbf{a})=\omega\left(g^{-1} A_{1}, \ldots, g^{-1} A_{k}\right),
$$

for any $\mathbf{a}=\left\{A_{1}, \ldots, A_{k}\right\} \in \mathcal{A}^{k}$. Equivalently $g \omega\left(g^{\prime} H_{n}\right)=\omega\left(g^{-1} g^{\prime} H_{n}\right)$ for every $g^{\prime} H_{n} \in G / H_{n}$. For each right $H_{n}$-invariant functions $f$ in $R U C(G)$ we denote the compact orbit closure of $f=\omega_{f}$ in $\Omega^{k}$ by $Y_{f}$.

First let us consider the case $n=1$, where $k=2$,

$\mathcal{A}^{2}=\left\{\mathbf{a}=\left\{A, A^{c}\right\}\right.$ : a partition of $C$ into two nonempty clopen sets $\}$, and

$$
H=H_{1}=\left\{g \in G: g I_{j}^{1}=I_{j}^{1}, j=1,2\right\} .
$$

Claim 13.1. There are exactly seven double cosets $\mathrm{HgH}, g \in G$.

Proof. For a partition $\left(A, A^{c}\right) \in \mathcal{A}^{2}$ exactly one of the following five possibilities holds: (1) $A=I_{1}^{1}$, (2) $A=I_{2}^{1}$, (3) $A \mp I_{1}^{1}$, (4) $A \subsetneq I_{2}^{1}$, (5) $A \supsetneq I_{1}^{1}$, (6) $A \supsetneq I_{2}^{1}$, (7) $A \cap I_{1}^{1} \neq \emptyset \neq A \cap I_{2}^{1}$, and $A^{c} \cap I_{1}^{1} \neq \emptyset \neq A^{c} \cap I_{2}^{1}$.

Clearly for any two partitions $\left(A, A^{c}\right),\left(B, B^{c}\right)$ we have $\left(B, B^{c}\right)=\left(h A, h A^{c}\right)$ for some $h \in H$ iff they belong to the same class. Our claim follows from the correspondence $G / H \cong \mathcal{A}^{2}$.

Define an element $\omega_{f} \in \Omega^{2}$ and the corresponding function $f \in U C(G)$ as follows:

$$
\omega\left(A, A^{c}\right)=j \quad \text { if }\left(A, A^{c}\right) \text { is of type }(j), \quad j=1, \ldots, 7,
$$


and $f(g)=\omega\left(g I_{1}^{1}, g I_{2}^{1}\right)$. Clearly $f$ is $H_{1}$-biinvariant and in particular an element of $U C(G)$. Let $X_{f}$ denote the (pointwise) orbit closure of $f$ in $R U C(G)$. Via the natural lift of $\Omega^{2}$ to $R U C(G)$ we can identify $X_{f}$ with $Y_{f}=\operatorname{cl}\left\{g \cdot \omega_{f}: g \in G\right\} \subset \Omega^{2}$.

Next consider a sequence of homeomorphisms $h_{n} \in G$ satisfying the conditions

(i) $h_{n}\left(I_{1}^{n}\right)=\left(I_{2^{n}}^{n}\right)^{c}$,

(ii) $h_{n}\left(\left(I_{1}^{n}\right)^{c}\right)=I_{2^{n}}^{n}$,

(iii) $h_{n}$ is order preserving.

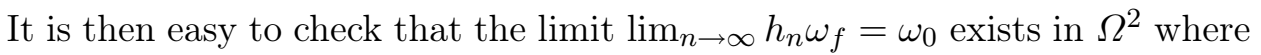
$\omega_{0}$ is defined by

$$
\omega_{0}\left(A, A^{c}\right)= \begin{cases}5 & \text { if } 0 \in A, \\ 4 & \text { if } 0 \notin A .\end{cases}
$$

Now for any $g \in G$ we have

$$
\left(g \cdot \omega_{0}\right)\left(A, A^{c}\right)=\omega_{0}\left(g^{-1} A, g^{-1} A^{c}\right)= \begin{cases}5 & \text { if } g(0) \in A, \\ 4 & \text { if } g(0) \notin A .\end{cases}
$$

For $x \in C$ set

$$
\left(\omega_{x}\right)\left(A, A^{c}\right)= \begin{cases}5 & \text { if } x \in A, \\ 4 & \text { if } x \notin A .\end{cases}
$$

Then for $g \in G$ we have $g \omega_{0}=\omega_{g 0}$. Moreover, setting $Y_{0}=\operatorname{cl}\left\{g \omega_{0}: g \in G\right\}$ $\subset \Omega^{2}$ we have $Y_{0}=G \omega_{0}$ and the map $\phi:(G, C) \rightarrow\left(G, Y_{0}\right)$ defined by $\phi(x)=\omega_{x}$ is an isomorphism of $G$-spaces. We get the following lemma.

Lemma 13.2. Let $Y_{0}=\operatorname{cl}\left\{g \omega_{0}: G \in G\right\}$ be the orbit closure of $\omega_{0}$ in $\Omega^{2}$. Then the $G$-space $\left(G, Y_{0}\right)$ is isomorphic to $(G, C)$, the natural action of $G=H(C)$ on the Cantor set $C$.

REMARK 13.3. An argument analogous to that of Lemma 13.2 will show that for every $n$ the number of $H_{n}$ double cosets is finite. As in the case of $S(\mathbb{N})$ in the previous section, this shows the well known fact that $G=H(C)$ is Roelcke precompact (see [48]).

In contrast to Theorem 12.2 we obtain the following result.

Theorem 13.4. For $G=H(C)$ we have $U C(G) \supsetneq S U C(G)$.

Proof. Consider the function

$$
f_{0}(g)=\omega_{0}\left(g^{-1} I_{1}^{1}, g^{-1} I_{2}^{1}\right)=g \omega_{0}\left(I_{1}^{1}, I_{2}^{1}\right)=\omega_{g 0}\left(I_{1}^{1}, I_{2}^{1}\right)
$$

and let $h_{n} \in G$ be defined as above. Let $u_{n}$ be a sequence of elements of $G$ which converges to $e \in G$ and for which $h_{n} u_{n} 0=2 / 3$. Then, as $h_{n} 0=0$ for every $n$, we have

$$
f_{0}\left(h_{n}\right)=\omega_{0}\left(h_{n}^{-1} I_{1}^{1}, h_{n}^{-1} I_{2}^{1}\right)=5
$$


but as $h_{n} u_{n} 0=2 / 3$,

$$
f_{0}\left(h_{n} u_{n}\right)=\omega_{0}\left(u_{n}^{-1} h_{n}^{-1} I_{1}^{1}, u_{n}^{-1} h_{n}^{-1} I_{2}^{1}\right)=\omega_{h_{n} u_{n} 0}\left(I_{1}^{1}, I_{2}^{1}\right)=4 .
$$

Thus $f_{0}$ is not left uniformly continuous. Since $f_{0} \in X_{f} \cong Y_{0}$, we conclude, by Theorem 4.12 , that $f$ is not a SUC function.

REMARK 13.5. A similar argument will show that any two points $a, b \in C$ are SUC-proximal for the $G$-space $(G, C)$. Thus this $G$-space is SUC-trivial by Lemma 8.2. Letting $F: Y_{0} \rightarrow\{4,5\} \subset \mathbb{R}$ be the evaluation function $F(\omega)=\omega\left(I_{1}^{1}, I_{2}^{1}\right)$, we observe that

$$
f_{0}(g)=\omega_{0}\left(g^{-1} I_{1}^{1}, g^{-1} I_{2}^{1}\right)=g \omega_{0}\left(I_{1}^{1}, I_{2}^{1}\right)=F\left(g \omega_{0}\right)=F(g \phi 0)=(F \circ \phi)(g 0) .
$$

Thus the function $f_{0}$ comes from the $G$ space $C$, via the continuous function $F \circ \phi: C \rightarrow \mathbb{R}$ and the point $0 \in C$. This is another way of showing that $f_{0}$ and hence also $f$ are not SUC.

Lemma 13.6. Let $\mathfrak{A} \subset R U C(G)$ be a closed $G$-invariant subalgebra containing the constants. Given $f \in \mathfrak{A}$ and $\varepsilon>0$ there exist an $n$ and an $H_{n}$-invariant function $\widehat{f} \in \mathfrak{A}$ with $\|f-\widehat{f}\|<\varepsilon$.

Proof. By right uniform continuity there exists an $n$ such that $\sup \{\mid f(h g)$ $-f(g) \mid: g \in G\}<\varepsilon$ for every $h \in H_{n}$. Set $\widehat{f}(g)=\sup _{h \in H_{n}} f(h g)$. This function is continuous because it is defined via the quotient map $G \rightarrow G / H_{n}$, where $G / H_{n}$ is discrete. Since for every finite set $\left\{h_{1}, \ldots, h_{t}\right\} \subset H_{n}$ the function

$$
f_{h_{1}, \ldots, h_{t}}(g)=\max _{1 \leq j \leq t} f\left(h_{j} g\right)
$$

is in $\mathfrak{A}$, Dini's theorem implies that also $\widehat{f}$ is an element of $\mathfrak{A}$.

REMARK 13.7.

(1) By Theorem 13.4 and Corollary 4.11 we deduce, in particular, that the algebra $U C(G)$ is not point-universal and the corresponding Roelcke compactification $G \rightarrow G^{U C}$ is not a right topological semigroup compactification of $G$. The same is true for $G:=H_{+}[0,1]$ because $U C(G) \neq S U C(G)$. This follows from Theorem 8.3 and Lemma 2.1.

(2) Using Lemma 13.6 one can try to determine, in analogy with the previous section, the structure of the universal WAP $G$-space, which is apparently much smaller than the Roelcke compactification of $G$.

TheOrem 13.8. The Polish group $G=H(C)$ of homeomorphisms of the Cantor set $C$ has the SUC-fpp.

Proof. Let $f$ be a minimal, SUC function (that is, $f$ is in $S U C(G)$ and $X_{f}$ is a minimal $G$-space). Let $\mathcal{A}$ be the smallest uniformly closed $G$-invariant 
algebra of $R U C(G)$ containing $f$ and the constant functions. As we have seen above, for every $\varepsilon>0$ there exist $n$ and $\widehat{f} \in R U C(G)$ such that, with

$$
H_{n}=\left\{g \in G: g I_{j}^{n}=I_{j}^{n}, \forall 1 \leq j \leq 2^{n}\right\},
$$

$\widehat{f}$ is $H$-biinvariant and $\|f-\widehat{f}\|<\varepsilon$. By Lemma 13.6, $\widehat{f}$ is still an element of the algebra $\mathcal{A} \cap S U C(G)$. It clearly suffices to show that $\widehat{f}$ is constant. For convenience of notation we will therefore assume that $f=\widehat{f}$. Let now $\omega_{f}\left(A_{1}, \ldots, A_{2^{n}}\right)$ be the corresponding configuration in $\Omega^{2^{n}}$. As we have seen, the function $f(g)=\omega_{f}\left(g^{-1} I_{1}^{n}, \ldots, g^{-1} I_{2^{n}}^{n}\right)$ has a finite range, and again an application of the sequence $h_{n}$ to $\omega_{f}$ will yield the limit configuration

$$
\omega_{0}\left(A_{1}, \ldots, A_{2^{n}}\right)= \begin{cases}a & \text { if } 0 \in A_{1}, \\ b & \text { if } 0 \notin A_{1},\end{cases}
$$

for some $a, b \in \mathbb{R}$. Since $f$ is a SUC function, $X_{f} \subset S U C(G)$, by Theorem 4.12, and the function $f_{0}(g)=\omega_{0}\left(g^{-1} \mathbf{I}^{n}\right)$, which is an element of $X_{f}$, is SUC. Since $f_{0}$ comes from the coset $G$-space $C$ (see Lemma 13.2 and Remark 13.5), Theorem 5.1 implies that it is constant (i.e. $a=b$ ). However, by assumption, $X_{f}$ is a minimal $G$-space and it follows that $f=f_{0}$ is constant.

\section{References}

[1] E. Akin, J. Auslander and K. Berg, When is a transitive map chaotic, in: Convergence in Ergodic Theory and Probability, de Gruyter, 1996, 25-40.

[2] - , - - , Almost equicontinuity and the enveloping semigroup, in: Topological Dynamics and Applications, Contemp. Math. 215, a volume in honor of R. Ellis, Amer. Math. Soc., 1998, 75-81.

[3] E. Akin and E. Glasner, Residual properties and almost equicontinuity, J. Anal. Math. 84 (2001), 243-286.

[4] W. Banaszczyk, Additive Subgroups of Topological Vector Spaces, Lecture Notes in Math. 1466, Springer, 1991.

[5] J. F. Berglund, H. D. Junghenn and P. Milnes, Compact Right Topological Semigroups and Generalizations of Almost Periodicity, Lecture Notes in Math. 663, Springer, 1978.

[6] R. D. Bourgin, Geometric Aspects of Convex Sets with the Radon-Nikodym Property, Lecture Notes in Math. 993, Springer, 1983.

[7] W. J. Davis, T. Figiel, W. B. Johnson and A. Pełczyński, Factoring weakly compact operators, J. Funct. Anal. 17 (1974), 311-327.

[8] R. Ellis, Equicontinuity and almost periodic functions, Proc. Amer. Math. Soc. 10 (1959), 637-643.

[9] R. Ellis and M. Nerurkar, Weakly almost periodic flows, Trans. Amer. Math. Soc. 313 (1989), 103-119.

[10] M. Fabian, Gâteaux Differentiability of Convex Functions and Topology. Weak Asplund Spaces, Canad. Math. Soc. Ser. Monogr. Adv. Texts, Wiley-Interscience, New York, 1997.

[11] E. Glasner, On minimal actions of Polish groups, Topology Appl. 85 (1998), 119-125. 
[12] E. Glasner, Ergodic Theory via Joinings, Math. Surveys Monogr. 101, Amer. Math. Soc., 2003.

[13] E. Glasner and M. Megrelishvili, Linear representations of hereditarily non-sensitive dynamical systems, Colloq. Math. 104 (2006), 223-283.

[14] E. Glasner, M. Megrelishvili and V. V. Uspenskij, On metrizable enveloping semigroups, Israel J. Math. 164 (2008), 317-332.

[15] E. Glasner and B. Weiss, Sensitive dependence on initial conditions, Nonlinearity 6 (1993), 1067-1075.

[16] —, - Locally equicontinuous dynamical systems, Colloq. Math. 84/85 (2000), part 2, 345-361.

[17] -, 一, Minimal actions of the group $S(\mathbb{Z})$ of permutations of the integers, Geom. Funct. Anal. 12 (2002), 964-988.

[18] - - - The universal minimal system for the group of homeomorphisms of the Cantor set, Fund. Math. 176 (2003), 277-289.

[19] E. Granirer and A. T. Lau, Invariant means on locally compact groups, Illinois J. Math. 15 (1971), 249-257.

[20] M. Gromov and V. D. Milman, A topological application of the isoperimetric inequality, Amer. J. Math. 105 (1983), 843-854.

[21] A. Grothendieck, Critères de compacité dans les espaces fonctionnelles généraux, Amer. J. Math. 74 (1952), 168-186.

[22] W. Herer and J. P. R. Christensen, On the existence of pathological submeasures and the construction of exotic topological groups, Math. Ann. 213 (1975), 203-210.

[23] J. E. Jayne and C. A. Rogers, Borel selectors for upper semicontinuous set-valued maps, Acta Math. 155 (1985), 41-79.

[24] M. Megrelishvili, A Tikhonov G-space not admitting a compact $G$-extension or a G-linearization, Russian Math. Surveys 43 (1988), 177-178.

[25] —, Free topological G-groups, New Zealand J. Math. 25 (1996), 59-72.

[26] - Fragmentability and continuity of semigroup actions, Semigroup Forum 57 (1998), 101-126.

[27] - Operator topologies and reflexive representability, in: Nuclear Groups and Lie Groups, Res. Exp. Math. 24, Heldermann, Berlin, 2001, 197-208.

[28] - Every semitopological semigroup compactification of the group $H_{+}[0,1]$ is trivial, Semigroup Forum 63 (2001), 357-370.

[29] -, Reflexively but not unitarily representable topological groups, Topology Proc. 25 (2002), 615-625.

[30] - Fragmentability and representations of flows, ibid. 27 (2003), 497-544; see also: www.math.biu.ac.il/ $\sim$ megereli.

[31] - Topological transformation groups: selected topics, in: Open Problems in Topology II, E. Pearl (ed.), Elsevier, 2007, 423-438.

[32] - Every topological group is a group retract of a minimal group, Topology Appl., to appear.

[33] T. Mitchell, Function algebras, means and fixed points, Trans. Amer. Math. Soc. 130 (1968), 117-126.

[34] J. von Neumann and E. P. Wigner, Minimally almost periodic groups, Ann. of Math. 41 (1940), 746-750.

[35] E. C. Nummela, On epimorphisms of topological groups, General Topology Appl. 9 (1978), 155-167.

[36] V. Pestov, Epimorphisms of Hausdorff groups by way of topological dynamics, New Zealand J. Math. 26 (1997), 257-262. 
[37] V. Pestov, On free actions, minimal flows and a problem by Ellis, Trans. Amer. Math. Soc. 350 (1998), 4149-4165.

[38] -, Topological groups: where to from here?, Topology Proc. 24 (1999), 421-502.

[39] —, The isometry group of the Urysohn space as a Lévy group, Topology Appl. 154 (2007), 2173-2184.

[40] -, Dynamics of Infinite-Dimensional Groups. The Ramsey-Dvoretzky-Milman Phenomenon, Univ. Lecture Ser. 40, Amer. Math. Soc., Providence, RI, 2006.

[41] W. Roelcke and S. Dierolf, Uniform Structures on Topological Groups and Their Quotients, McGraw-Hill, New York, 1981.

[42] C. Rosendal and S. Solecki, Automatic continuity of homomorphisms and fixed point on metric compacta, Israel J. Math. 162 (2007), 349-371.

[43] S. Teleman, Sur la représentation linéaire des groupes topologiques, Ann. Sci. École Norm. Sup. 74 (1957), 319-339.

[44] V. V. Uspenskij, The epimorphism problem for Hausdorff topological groups, Topology Appl. 57 (1994), 287-294.

[45] -, On subgroups of minimal topological groups, in: Proc. Int. Workshop on the Urysohn Metric Space (Ben-Gurion Univ., 2006), Topology Appl., to appear.

[46] —, The Roelcke compactification of unitary groups, in: Abelian Groups, Module Theory, and Topology, in honor of Adalberto Orsatti (D. Dikranjan and L. Salce, eds.), Lecture Notes in Pure Appl. Math. 201, Dekker, New York, 1998, 411-419.

[47] -, Compactifications of topological groups, in: Proc. Ninth Prague Topological Symposium (Prague, 2001), P. Simon (ed.), Topology Atlas (electronic publication), 2002, 331-346; arXiv:math.GN/0204144.

[48] - , The Roelcke compactification of groups of homeomorphisms, Topology Appl. 111 (2001), 195-205.

[49] W. A. Veech, Weakly almost periodic functions on semisimple Lie groups, Monatsh. Math. 88 (1979), 55-68.

[50] J. de Vries, Equivariant embeddings of G-spaces, in: J. Novak (ed.), General Topology and its Relations to Modern Analysis and Algebra IV, Part B, Prague, 1977, 485-493.

[51] —, Elements of Topological Dynamics, Kluwer, 1993.

Department of Mathematics

Tel-Aviv University

Ramat Aviv, Israel

E-mail: glasner@math.tau.ac.il

http://www.math.tau.ac.il/ glasner
Department of Mathematics Bar-Ilan University 52900 Ramat-Gan, Israel

E-mail: megereli@math.biu.ac.il http://www.math.biu.ac.il/ ${ }^{\sim}$ megereli

Received 2 January 2007;

in revised form 26 March 2008 